\title{
Casimir effect for scalar current densities in topologically nontrivial spaces
}

\author{
S. Bellucci ${ }^{1, a}$, A. A. Saharian ${ }^{2, b}$, N. A. Saharyan ${ }^{2}$ \\ ${ }^{1}$ INFN, Laboratori Nazionali di Frascati, Via Enrico Fermi 40, 00044 Frascati, Italy \\ 2 Department of Physics, Yerevan State University, 1 Alex Manoogian Street, 0025 Yerevan, Armenia
}

Received: 6 August 2015 / Accepted: 11 August 2015 / Published online: 22 August 2015

(c) The Author(s) 2015. This article is published with open access at Springerlink.com

\begin{abstract}
We evaluate the Hadamard function and the vacuum expectation value (VEV) of the current density for a charged scalar field, induced by flat boundaries in spacetimes with an arbitrary number of toroidally compactified spatial dimensions. The field operator obeys the Robin conditions on the boundaries and quasiperiodicity conditions with general phases along compact dimensions. In addition, the presence of a constant gauge field is assumed. The latter induces Aharonov-Bohm-type effect on the VEVs. There is a region in the space of the parameters in Robin boundary conditions where the vacuum state becomes unstable. The stability condition depends on the lengths of compact dimensions and is less restrictive than that for background with trivial topology. The vacuum current density is a periodic function of the magnetic flux, enclosed by compact dimensions, with the period equal to the flux quantum. It is explicitly decomposed into the boundary-free and boundary-induced contributions. In sharp contrast to the VEVs of the field squared and the energy-momentum tensor, the current density does not contain surface divergences. Moreover, for Dirichlet condition it vanishes on the boundaries. The normal derivative of the current density on the boundaries vanish for both Dirichlet and Neumann conditions and is nonzero for general Robin conditions. When the separation between the plates is smaller than other length scales, the behavior of the current density is essentially different for non-Neumann and Neumann boundary conditions. In the former case, the total current density in the region between the plates tends to zero. For Neumann boundary condition on both plates, the current density is dominated by the interference part and is inversely proportional to the separation.
\end{abstract}

\footnotetext{
a e-mail: bellucci@lnf.infn.it

be-mail: saharian@ysu.am
}

\section{Introduction}

In a number of physical problems one needs to consider the model in the background of manifolds with boundaries on which the dynamical variables obey some prescribed boundary conditions. In quantum field theory, the imposition of boundary conditions on the field operator gives rise to a number of physical consequences. The Casimir effect is among the most interesting phenomena of this kind (for reviews see [1-6]). It arises due to the modification of the quantum fluctuations of a field by boundary conditions and plays an important role in different fields of physics, from microworld to cosmology. The boundary conditions in the Casimir effect may have different physical natures and can be divided into two main classes. In the first one, the constraints are induced by the presence of boundaries, like macroscopic bodies in QED, interfaces separating different phases of a physical system, extended topological defects, horizons in gravitational physics, branes in high-energy theories with extra dimensions and in string theories. In the corresponding models the field operator obeys the boundary condition on some spacelike surfaces (static or dynamical). The original problem with two conducting plates, discussed by Casimir in 1948 [7], belongs to this class. Since the original research by Casimir, much theoretical and experimental work has been done on this problem for various types of bulk and boundary geometries. Different methods have been developed including direct mode-summation and the zeta function techniques, semiclassical methods, the optical approach, worldline numerics, the path integral approach, methods based on scattering theory, and numerical methods based on an evaluation of the stress tensor via the fluctuation-dissipation theorem. The recent high precision measurements of the Casimir force allow for an accurate comparison between the experimental results and theoretical predictions.

In the second class, the boundary conditions on the field operator are induced by the nontrivial topology of the space. 
The changes in the properties of the vacuum state generated by this type of conditions are referred to as the topological Casimir effect. The importance of this effect is motivated by that the presence of compact dimensions is an inherent feature in many high-energy theories of fundamental physics, in cosmology and in condensed matter physics. In particular, supergravity and superstring theories are formulated in spacetimes having extra compact dimensions. The compactified higher-dimensional models provide a possibility for the unification of known interactions. Models of a compact universe with nontrivial topology may also play an important role by providing proper initial conditions for inflation in the early stages of the Universe expansion [8]. In condensed matter physics, a number of planar systems in the low-energy sector are described by an effective field theory. The compactification of these systems leads to the change in the ground state energy, which is the analog of the topological Casimir effect. A well-known example of this type of systems is a graphene sheet. In the long wavelength limit, the dynamics of the quasiparticles for the electronic subsystem is described in terms of the Dirac-like theory in two-dimensional space (see Refs. $[9,10]$ ). The corresponding effective three-dimensional relativistic field theory, in addition to Dirac fermions, involves scalar and gauge fields (see [11,12] and references therein). The single-walled carbon nanotubes are generated by rolling up a graphene sheet to form a cylinder and for the corresponding Dirac model one has the spatial topology $R^{1} \times S^{1}$. For another class of graphene-made structures, called toroidal carbon nanotubes, the background topology is a two-dimensional torus, $T^{2}$.

Many authors have investigated the Casimir energies and stresses associated with the presence of compact dimensions (for reviews see Refs. [1-6,13-18]). In higher-dimensional models the Casimir energy of bulk fields induces an effective potential for the compactification radius. This has been used as a stabilization mechanism for the corresponding moduli fields and as a source for dynamical compactification of the extra dimensions during the cosmological evolution. The Casimir effect has also been considered as a possible origin for the dark energy in both Kaluza-Klein-type and braneworld models [19-27]. Extra-dimensional theories with low-energy compactification scale predict Yukawa-type corrections to Newton's gravitational law and the measurements of the Casimir forces between macroscopic bodies provide a sensitive test for constraining the parameters of the corresponding long-range interactions [28-32]. The influence of extra compactified dimensions on the Casimir effect in the classical configuration of two parallel plates has been recently discussed for scalar [33-40], electromagnetic [4145] and fermionic [46-48] fields.

The vast majority of the works on the influence of the compactification on the properties of the quantum vacuum in the Casimir effect has been concerned with global quantities such as the force or the total energy. More detailed information on the vacuum fluctuations is contained in the local characteristics. Among the most important local quantities, because of their close connection with the structure of spacetime, are the vacuum expectation values (VEVs) of the vacuum energy density and stresses. For charged fields, another important characteristic is the VEV of the current density. Due to the global nature of the vacuum, this VEV carries information on both global and local properties of the vacuum state. Besides, the VEV of the current density appears as a source of the electromagnetic field in semiclassical Maxwell equations, and, hence, it is needed in modeling a self-consistent dynamics involving the electromagnetic field.

In models with a nontrivial topology, the nonzero current densities in the vacuum state may appear as a consequence of quasiperiodicity conditions along compact dimensions or by the presence of gauge field fluxes enclosed by these dimensions. Note that the gauge field fluxes in higher-dimensional models will also generate a potential for moduli fields and this provides another mechanism for moduli stabilization (for a review see [49]). The VEV of the fermionic current density in spaces with toroidally compactified dimensions has been considered in [50]. In the special case of a two-dimensional space, applications are found in the electrons in cylindrical and toroidal carbon nanotubes, described within the framework of the effective field theory in terms of Dirac fermions. The vacuum currents for charged fields in de Sitter and antide Sitter spacetimes with toroidally compact spatial dimensions are investigated in [51,52]. Finite temperature effects on the charge density and on the current densities along compact dimensions have been discussed in [53] and [54] for scalar and fermionic fields, respectively. The changes in the fermionic vacuum currents induced by the presence of parallel plane boundaries, with the bag boundary conditions, are investigated in [55].

In the present paper we consider the effect of two parallel plane boundaries on the vacuum expectation value of the current density for a charged scalar field in background spacetime with spatial topology $R^{p+1} \times T^{q}$, where $T^{q}$ stands for a $q$-dimensional torus. The organization of the paper is as follows. In the next section the geometry of the problem is described and the Hadamard function is evaluated in the region between the plates for general Robin boundary conditions. By using the expression for the Hadamard function, in Sect. 3, we evaluate the current density in the geometry of a single plate. The corresponding asymptotics are discussed in various limiting cases and numerical results are presented. In Sect. 4 the current density is investigated in the region between two plates. The main results of the paper are summarized in Sect. 5. An alternative representation of the Hadamard function is given in "Appendix". 


\section{Formulation of the problem and the Hadamard function}

We consider $(D+1)$-dimensional flat spacetime with spatial topology $R^{p+1} \times T^{q}, p+q+1=D$ (for a review of quantum field-theoretical effects in toroidal topology see Ref. [18]). The set of Cartesian coordinates in the subspace $R^{p+1}$ will be denoted by $\mathbf{x}_{p+1}=\left(x^{1}, \ldots, x^{p+1}\right)$ and the corresponding coordinates on the torus by $\mathbf{x}_{q}=\left(x^{p+2}, \ldots, x^{D}\right)$. If $L_{l}$ is the length of the $l$ th compact dimension then one has $-\infty<$ $x^{l}<\infty$ for $l=1, . ., p$, and $0 \leqslant x^{l} \leqslant L_{l}$ for $l=p+$ $2, \ldots, D$. Our main interest in this paper is the VEV of the current density for a quantum scalar field $\varphi(x)$ with the mass $m$ and charge $e$. The equation for the field operator reads

$$
\left(g^{\mu v} D_{\mu} D_{v}+m^{2}\right) \varphi=0
$$

where $g^{\mu \nu}=\operatorname{diag}(1,-1, \ldots,-1), D_{\mu}=\partial_{\mu}+i e A_{\mu}$, and $A_{\mu}$ is the vector potential for a classical gauge field. We assume the presence of two parallel flat boundaries ${ }^{1}$ placed at $x^{p+1}=a_{1}$ and $x^{p+1}=a_{2}$, on which the field obeys Robin boundary conditions

$\left(1+\beta_{j} n_{j}^{\mu} D_{\mu}\right) \varphi(x)=0, \quad x^{p+1} \equiv z=a_{j}$,

with constant coefficients $\beta_{j}, j=1,2$, and with $n_{j}^{\mu}$ being the inward pointing normal to the boundary at $x^{p+1}=a_{j}$. Here, for the further convenience we have introduced a special notation $z=x^{p+1}$ for the $(p+1)$ th spatial dimension. Note that Robin boundary conditions in the form (2.2) are gauge invariant (for the discussion of various types of gauge invariant boundary conditions see $[56,57])$. In what follows we will consider the region between the plates, $a_{1} \leqslant z \leqslant a_{2}$. For this region one has $n_{j}^{\mu}=(-1)^{j-1} \delta_{p+1}^{\mu}$. The expressions for the VEVs in the regions $z \leqslant a_{1}$ and $z \geqslant a_{2}$ are obtained by the limiting transitions. The results for Dirichlet and Neumann boundary conditions are obtained from those for the condition (2.2) in the limits $\beta_{j} \rightarrow 0$ and $\beta_{j} \rightarrow \infty$, $A_{\mu}=0$, respectively. Robin type conditions appear in a variety of situations, including the considerations of vacuum effects for a confined charged scalar field in external fields [58], gauge field theories, quantum gravity and supergravity [56,57,59], braneworld models [60-62] and in a class of models with boundaries separating the spatial regions with different gravitational backgrounds [63-65]. In some geometries, these conditions may be useful for depicting the finite penetration of the field into the boundary with the "skindepth" parameter related to the coefficient $\beta_{j}$. It is interesting to note that the quantum scalar field constrained by the Robin condition on the boundary of cavity violates the Bekenstein

\footnotetext{
${ }^{1}$ In analogy with the standard Casimir effect, in the discussion below we will refer the boundaries as plates.
}

entropy-to-energy bound near certain points in the space of the parameter $\beta_{j}$ [66].

In addition to the boundary conditions on the plates, for the theory to be completely defined, we should also specify the periodicity conditions along the compact dimensions. Different conditions correspond to topologically inequivalent field configurations $[67,68]$. Here, we consider generic quasiperiodicity conditions,

$$
\begin{aligned}
& \varphi\left(t, x^{1}, \ldots, x^{l}+L_{l}, \ldots, x^{D}\right) \\
& \quad=e^{i \alpha_{l}} \varphi\left(t, x^{1}, \ldots, x^{l}, \ldots, x^{D}\right),
\end{aligned}
$$

with constant phases $\alpha_{l}, l=p+2, \ldots, D$. The special cases of the condition (2.3) with $\alpha_{l}=0$ and $\alpha_{l}=\pi$ correspond to the most frequently discussed cases of untwisted and twisted scalar fields, respectively. As it will be seen below, one of the effects of nontrivial phases in (2.3) is the appearance of nonzero vacuum currents along compact dimensions (for a discussion of physical effects of phases in periodicity conditions along compact dimensions see [69-75] and references therein).

For a scalar field, the operator of the current density is given by the expression

$j_{\mu}(x)=i e\left[\varphi^{+}(x) D_{\mu} \varphi(x)-\left(D_{\mu} \varphi(x)\right)^{+} \varphi(x)\right]$,

$l=0,1, \ldots, D$. Its VEV is obtained from the Hadamard function

$G\left(x, x^{\prime}\right)=\left\langle 0\left|\varphi(x) \varphi^{+}\left(x^{\prime}\right)+\varphi^{+}\left(x^{\prime}\right) \varphi(x)\right| 0\right\rangle$,

with $|0\rangle$ being the vacuum state, by using the formula

$$
\begin{aligned}
\left\langle 0\left|j_{\mu}(x)\right| 0\right\rangle & \equiv\left\langle j_{\mu}(x)\right\rangle \\
& =\frac{i}{2} e \lim _{x^{\prime} \rightarrow x}\left(\partial_{\mu}-\partial_{\mu}^{\prime}+2 i e A_{\mu}\right) G\left(x, x^{\prime}\right) .
\end{aligned}
$$

In the discussion below we will assume a constant gauge field $A_{\mu}$. Though the corresponding field strength vanishes, the nontrivial topology of the background spacetime leads to the Aharonov-Bohm-like effects on physical observables. In the case of a constant gauge field $A_{\mu}$, the latter can be excluded from the field equation and from the expression for the VEV of the current density by the gauge transformation $A_{\mu}=A_{\mu}^{\prime}+\partial_{\mu} \chi, \varphi(x)=e^{-i e \chi} \varphi^{\prime}(x)$, with the function $\chi=A_{\mu} x^{\mu}$. In the new gauge one has $A_{\mu}^{\prime}=0$. However, unlike to the case of a trivial topology, here the constant vector potential does not completely disappear from the problem. It appears in the periodicity conditions for the new field operator:

$$
\begin{aligned}
& \varphi^{\prime}\left(t, x^{1}, \ldots, x^{l}+L_{l}, \ldots, x^{D}\right) \\
& \quad=e^{i \tilde{\alpha}_{l}} \varphi^{\prime}\left(t, x^{1}, \ldots, x^{l}, \ldots, x^{D}\right),
\end{aligned}
$$

where now the phases are given by the expression

$\tilde{\alpha}_{l}=\alpha_{l}+e A_{l} L_{l}$. 
In the discussion below we shall consider the problem in the gauge $\left(\varphi^{\prime}(x), A_{\mu}^{\prime}=0\right)$ omitting the prime. For this gauge, in (2.1), (2.2), (2.4) one has $D_{\mu}=\partial_{\mu}$ and in the expressions (2.6) the term with the vector potential is absent.

From the discussion above it follows that in the problem at hand the presence of a constant gauge field is equivalent to the shift in the phases of the periodicity conditions along compact dimensions. The shift in the phase is expressed in terms of the magnetic flux $\Phi_{l}$ enclosed by the $l$ th compact dimension as

$e A_{l} L_{l}=-e \mathbf{A}_{l} L_{l}=-2 \pi \Phi_{l} / \Phi_{0}$,

where $\Phi_{0}=2 \pi / e$ is the flux quantum and $\mathbf{A}_{l}$ is the $l$ th component of the spatial vector $\mathbf{A}=\left(-A_{1}, \ldots,-A_{D}\right)$. In the discussion below the physical effects of a constant gauge field will appear through the phases $\tilde{\alpha}_{l}$. In particular, the VEVs of physical observables are periodic functions of these phases with the period $2 \pi$. In terms of the magnetic flux, this corresponds to the periodicity of the VEVs, as functions of the magnetic flux, with the period equal to the flux quantum.

For the evaluation of the Hadamard function in (2.6) we shall use the mode-sum formula

$G\left(x, x^{\prime}\right)=\sum_{\mathbf{k}} \sum_{s= \pm} \varphi_{\mathbf{k}}^{(s)}(x) \varphi_{\mathbf{k}}^{(s) *}\left(x^{\prime}\right)$,

where $\varphi_{\mathbf{k}}^{( \pm)}(x)$ form a complete set of normalized positiveand negative-energy solutions to the classical field equation obeying the boundary conditions of the model. In the region between the plates, introducing the wave vectors $\mathbf{k}_{p}=\left(k_{1}, \ldots, k_{p}\right)$ and $\mathbf{k}_{q}=\left(k_{p+2}, \ldots, k_{D}\right)$, these mode functions can be written in the form

$\varphi_{\mathbf{k}}^{( \pm)}(x)=C_{\mathbf{k}} \cos \left[k_{p+1}\left(z-a_{j}\right)+\gamma_{j}\left(k_{p+1}\right)\right] e^{i \mathbf{k}_{\|} \cdot \mathbf{x}_{\|} \mp i \omega_{\mathbf{k}} t}$,

where $\mathbf{k}_{\|}=\left(\mathbf{k}_{p}, \mathbf{k}_{q}\right), \mathbf{k}=\left(\mathbf{k}_{p}, k_{p+1}, \mathbf{k}_{q}\right), \omega_{\mathbf{k}}=\sqrt{\mathbf{k}^{2}+m^{2}}$, and $\mathbf{x}_{\|}$stands for the coordinates parallel to the plates. For the momentum components along the dimensions $x^{i}$, $i=1, \ldots, p$, one has $-\infty<k_{i}<+\infty$, whereas the components along the compact dimensions are quantized by the periodicity conditions (2.7):

$k_{l}=\left(2 \pi n_{l}+\tilde{\alpha}_{l}\right) / L_{l}, \quad n_{l}=0, \pm 1, \pm 2, \ldots$,

with $l=p+2, \ldots, D$. We will denote by $\omega_{0}$ the smallest value for the energy in the compact subspace, $\sqrt{\mathbf{k}_{q}^{2}+m^{2}} \geqslant$ $\omega_{0}$. Assuming that $\left|\tilde{\alpha}_{l}\right| \leqslant \pi$, we have

$\omega_{0}=\sqrt{\sum_{l=p+2}^{D} \tilde{\alpha}_{l}^{2} / L_{l}^{2}+m^{2}}$.

This quantity can be considered as the effective mass for the field quanta.
Now we should impose on the modes (2.11) the boundary conditions (2.2) with $D_{\mu}=\partial_{\mu}$. From the boundary condition on the plate at $z=a_{j}$, for the function $\gamma_{j}\left(k_{p+1}\right)$ in (2.11) one gets

$e^{2 i \gamma_{j}\left(k_{p+1}\right)}=\frac{i k_{p+1} \beta_{j}(-1)^{j}+1}{i k_{p+1} \beta_{j}(-1)^{j}-1}$.

From the boundary condition on the second plate it follows that the eigenvalues for $k_{p+1}$ are solutions of the equation

$e^{2 i y}=\frac{1+i b_{2} y}{1-i b_{2} y} \frac{1+i b_{1} y}{1-i b_{1} y}$,

where

$y=k_{p+1} a, b_{j}=\beta_{j} / a$,

and $a=a_{2}-a_{1}$ is the separation between the plates. Equation (2.15) can also be written in the form

$$
\left(1-b_{1} b_{2} y^{2}\right) \sin y-\left(b_{2}+b_{1}\right) y \cos y=0 \text {. }
$$

Unlike to the cases of Dirichlet and Neumann conditions, for Robin boundary condition the eigenvalues of $k_{p+1}$ are given implicitly, as solutions of the transcendental equation (2.17). This equation has an infinite number of positive roots which will be denoted by $y=\lambda_{n}, n=1,2, \ldots$, and for the corresponding eigenvalues of $k_{p+1}$ one has $k_{p+1}=\lambda_{n} / a$. For $b_{j} \leqslant 0$ or $\left\{b_{1}+b_{2} \geqslant 1, b_{1} b_{2} \leqslant 0\right\}$ there are no other roots in the right-half plane of a complex variable $y, \operatorname{Re} y \geqslant 0$ (see [76]). In the remaining region of the plane $\left(b_{1}, b_{2}\right)$, Eq. (2.17) has purely imaginary roots $\pm i y_{l}, y_{l}>0$. Depending on the values of $b_{j}$, the number of $y_{l}$ can be one or two. In the presence of purely imaginary roots, under the condition $\omega_{0}<y_{l}$, there are modes of the field for which the energy $\omega_{\mathbf{k}}$ becomes imaginary. This would lead to the instability of the vacuum state. In the discussion below we will assume that $\omega_{0}>y_{l}$. Note that in the corresponding problem on background of spacetime with trivial topology the stability condition is written as $m>y_{l}$. Now, by taking into account that $\omega_{0}>m$, we conclude that the compactification, in general, enlarges the stability range in the space of parameters of Robin boundary conditions.

The coefficient $C_{\mathbf{k}}$ in (2.11) is found from the orthonormalization condition

$$
\begin{aligned}
& \int d^{D} x \varphi_{\mathbf{k}}^{(\lambda)}(x) \varphi_{\mathbf{k}^{\prime}}^{\left(\lambda^{\prime}\right) *}(x) \\
& =\frac{\delta_{\lambda \lambda^{\prime}}}{2 \omega_{\mathbf{k}}} \delta\left(\mathbf{k}_{p}-\mathbf{k}_{p}^{\prime}\right) \delta_{n n^{\prime}} \delta_{n_{p+2}, n_{p+2}^{\prime}} \ldots \delta_{n_{D}, n_{D}^{\prime}}
\end{aligned}
$$

where the integration over $x^{p+1}$ goes in the region between the plates. Substituting the functions (2.11), one gets

$$
\left|C_{\mathbf{k}}\right|^{2}=\frac{\left\{1+\cos \left[y+2 \tilde{\gamma}_{j}(y)\right] \sin (y) / y\right\}^{-1}}{(2 \pi)^{p} a V_{q} \omega_{\mathbf{k}}},
$$


where $y$ is a root of Eq. (2.17) and $V_{q}=L_{p+1} \ldots L_{D}$ is the volume of the compact subspace. The function $\tilde{\gamma}_{j}(y)$ is defined by the relation

$e^{2 i \tilde{\gamma}_{j}(y)}=\frac{i y b_{j}-1}{i y b_{j}+1}$.

First we shall consider the case when all the roots of (2.17) are real and $y=\lambda_{n}$.

Having the complete set of normalized mode functions, the mode-sum (2.10) for the Hadamard function is written in the form

$$
\begin{aligned}
G\left(x, x^{\prime}\right)= & \frac{1}{a V_{q}} \int \frac{\mathrm{d} \mathbf{k}_{p}}{(2 \pi)^{p}} \sum_{\mathbf{n}_{q}} \sum_{n=1}^{\infty} \frac{1}{\omega_{\mathbf{k}}} g_{j}\left(z, z^{\prime}, \lambda_{n} / a\right) \\
& \times \frac{\lambda_{n} \cos \left(\omega_{\mathbf{k}} \Delta t\right) e^{i \mathbf{k}_{p} \cdot \Delta \mathbf{x}_{p}+i \mathbf{k}_{q} \cdot \Delta \mathbf{x}_{q}}}{\lambda_{n}+\cos \left[\lambda_{n}+2 \tilde{\gamma}_{j}\left(\lambda_{n}\right)\right] \sin \lambda_{n}},
\end{aligned}
$$

where $\Delta \mathbf{x}_{p}=\mathbf{x}_{p}-\mathbf{x}_{p}^{\prime}, \Delta \mathbf{x}_{q}=\mathbf{x}_{q}-\mathbf{x}_{q}^{\prime}, \Delta t=t-t^{\prime}$, and $\mathbf{n}_{q}=\left(n_{p+2}, \ldots, n_{D}\right),-\infty<n_{l}<+\infty$. In (2.21), the energy for the mode with a given $\mathbf{k}$ is written as

$\omega_{\mathbf{k}}=\sqrt{\mathbf{k}_{p}^{2}+\lambda_{n}^{2} / a^{2}+\omega_{\mathbf{n}_{q}}^{2}}$,

and

$\omega_{\mathbf{n}_{q}}=\sqrt{\mathbf{k}_{q}^{2}+m^{2}}, \mathbf{k}_{q}^{2}=\sum_{l=p+2}^{D}\left(\frac{2 \pi n_{l}+\tilde{\alpha}_{l}}{L_{l}}\right)^{2}$.

Here and in what follows we use the notation

$g_{j}\left(z, z^{\prime}, u\right)=\cos (u \Delta z)+\frac{1}{2} \sum_{s= \pm 1} e^{s i y\left|z+z^{\prime}-2 a_{j}\right|} \frac{i u \beta_{j}-s}{i u \beta_{j}+s}$.

Note that $g_{j}\left(z, z^{\prime},-y\right)=g_{j}\left(z, z^{\prime}, y\right)$ and $g_{j}\left(z, z^{\prime}, 0\right)=0$.

In (2.21), the eigenvalues $\lambda_{n}$ are given implicitly and this expression is not convenient for the evaluation of the VEVs. In order to obtain an expression in which the explicit knowledge of $\lambda_{n}$ is not required, we apply to the series over $n$ the Abel-Plana-type summation formula $[76,77]$

$$
\begin{gathered}
\sum_{n=1}^{\infty} \frac{\pi \lambda_{n} f\left(\lambda_{n}\right)}{\lambda_{n}+\cos \left[\lambda_{n}+2 \tilde{\gamma}_{j}\left(\lambda_{n}\right)\right] \sin \lambda_{n}} \\
=-\frac{\pi f(0) / 2}{1-b_{2}-b_{1}}+\int_{0}^{\infty} d u f(u) \\
+i \int_{0}^{\infty} d u \frac{f(i u)-f(-i u)}{c_{1}(u) c_{2}(u) e^{2 u}-1},
\end{gathered}
$$

where, for further convenience, the notation

$c_{j}(u)=\frac{b_{j} u-1}{b_{j} u+1}$

is introduced. In (2.25) we have assumed that $b_{j} \leqslant 0$. The changes in the evaluation procedure in the case $b_{j}>0$ will be discussed below. For the series in (2.21), we take in the summation formula

$f\left(\lambda_{n}\right)=\frac{\cos \left(\omega_{\mathbf{k}} \Delta t\right)}{\omega_{\mathbf{k}}} g_{j}\left(z, z^{\prime}, \lambda_{n} / a\right)$.

Note that $f(0)=0$ and the first term in the right-hand side of (2.25) is absent.

The use of the summation formula (2.25) with (2.27) allows us to write the Hadamard function in the decomposed form

$$
\begin{aligned}
& G\left(x, x^{\prime}\right)=G_{j}\left(x, x^{\prime}\right) \\
& +\frac{2}{\pi V_{q}} \int \frac{\mathrm{d} \mathbf{k}_{p}}{(2 \pi)^{p}} \sum_{\mathbf{n}_{q}} \int_{a \omega_{\mathbf{k}_{\|}}}^{\infty} d u g_{j}\left(z, z^{\prime}, i u / a\right) \\
& \times \frac{e^{i \mathbf{k}_{p} \cdot \Delta \mathbf{x}_{p}+i \mathbf{k}_{q} \cdot \Delta \mathbf{x}_{q}}}{c_{1}(u) c_{2}(u) e^{2 u}-1} \frac{\cos h\left(\Delta t \sqrt{u^{2} / a^{2}-\omega_{\mathbf{k}_{\|}}}\right)}{\sqrt{u^{2}-a^{2} \omega_{\mathbf{k}_{\|}}}},
\end{aligned}
$$

where $\omega_{\mathbf{k}_{\|}}=\sqrt{\mathbf{k}_{p}^{2}+\omega_{\mathbf{n}_{q}}^{2}}$. Here, the part

$$
\begin{gathered}
G_{j}\left(x, x^{\prime}\right)=\frac{1}{\pi V_{q}} \int \frac{\mathrm{d} \mathbf{k}_{p}}{(2 \pi)^{p}} \sum_{\mathbf{n}_{q}} e^{i \mathbf{k}_{p} \cdot \Delta \mathbf{x}_{p}+i \mathbf{k}_{q} \cdot \Delta \mathbf{x}_{q}} \\
\times \int_{0}^{\infty} d k_{p+1} \frac{\cos \left(\omega_{\mathbf{k}} \Delta t\right)}{\omega_{\mathbf{k}}} g_{j}\left(z, z^{\prime}, k_{p+1}\right),
\end{gathered}
$$

comes from the first integral in the right-hand side of (2.25) and corresponds to the Hadamard function in the geometry of a single plate at $x^{p+1}=a_{j}$ when the second plate is absent. This function is further decomposed by taking into account that the part in (2.24) coming from the first term in the right-hand side of (2.24),

$$
\begin{aligned}
& G_{0}\left(x, x^{\prime}\right)=\frac{1}{V_{q}} \int \frac{\mathrm{d} \mathbf{k}_{p+1}}{(2 \pi)^{p+1}} \\
& \times \sum_{\mathbf{n}_{q}} e^{i \mathbf{k}_{p+1} \cdot \Delta \mathbf{x}_{p+1}+i \mathbf{k}_{q} \cdot \Delta \mathbf{x}_{q}} \frac{\cos \left(\omega_{\mathbf{k}} \Delta t\right)}{\omega_{\mathbf{k}}},
\end{aligned}
$$

is the Hadamard function for the boundary-free geometry. After the integration over the components of the momentum along non-compactified dimensions, this function can be presented in the form

$$
\begin{aligned}
& G_{0}\left(x, x^{\prime}\right)=\frac{2 V_{q}^{-1}}{(2 \pi)^{p / 2+1}} \\
& \quad \times \sum_{\mathbf{n}_{q}} e^{i \mathbf{k}_{q} \cdot \Delta \mathbf{x}_{q}} \omega_{\mathbf{n}_{q}}^{p} f_{p / 2}\left(\omega_{\mathbf{n}_{q}} \sqrt{\left|\Delta \mathbf{x}_{p+1}\right|^{2}-(\Delta t)^{2}}\right),
\end{aligned}
$$

with the notations

$$
f_{v}(x)=K_{v}(x) / x^{v},
$$

where $K_{v}(x)$ is the Macdonald function. 
Consequently, the Hadamard function in the geometry of a single plate is written as

$$
\begin{aligned}
& G_{j}\left(x, x^{\prime}\right)=G_{0}\left(x, x^{\prime}\right) \\
& +\frac{1}{2 \pi V_{q}} \int \frac{\mathrm{d} \mathbf{k}_{p}}{(2 \pi)^{p}} \sum_{\mathbf{n}_{q}} e^{i \mathbf{k}_{p} \cdot \Delta \mathbf{x}_{p}+i \mathbf{k}_{q} \cdot \Delta \mathbf{x}_{q}} \\
& \quad \times \sum_{s= \pm 1} \int_{0}^{\infty} \mathrm{d} k_{p+1} \frac{\cos \left(\omega_{\mathbf{k}} \Delta t\right)}{\omega_{\mathbf{k}}} e^{s i k_{p+1}\left|z+z^{\prime}-2 a_{j}\right|} \frac{i k_{p+1} \beta_{j}-s}{i k_{p+1} \beta_{j}+s},
\end{aligned}
$$

where the second term in the right-hand side is induced by the presence of the plate at $x^{p+1}=a_{j}$. For the further transformation of the boundary-induced part in (2.33) we rotate the integration contour over $k_{p+1}$ by the angle $s \pi / 2$. In the summation over $s$ the integrals over the intervals $\left(0, \pm i \omega_{\mathbf{k}_{\|}}\right)$ cancel each other and we get

$$
\begin{aligned}
& G_{j}\left(x, x^{\prime}\right)=G_{0}\left(x, x^{\prime}\right) \\
& +\frac{1}{\pi V_{q}} \int \frac{d \mathbf{k}_{p}}{(2 \pi)^{p}} \sum_{\mathbf{n}_{q}} e^{i \mathbf{k}_{p} \cdot \Delta \mathbf{x}_{p}+i \mathbf{k}_{q} \cdot \Delta \mathbf{x}_{q}} \\
& \times \int_{\omega_{\mathbf{k}_{\|}}}^{\infty} d u \frac{\cos h\left(\Delta t \sqrt{u^{2}-\omega_{\mathbf{k}_{\|}}^{2}}\right)}{\sqrt{u^{2}-\omega_{\mathbf{k}_{\|}^{2}}^{2}}} \frac{u \beta_{j}+1}{u \beta_{j}-1} e^{-u\left|z+z^{\prime}-2 a_{j}\right|} .
\end{aligned}
$$

This expression is well suited for the investigation of the current density. With the representation (2.34), the Hadamard function in the region between the plates, given by (2.28), is decomposed into the boundary-free, single plateinduced and second-plate-induced contributions. An alternative expression for the Hadamard function is obtained in the "Appendix".

In deriving (2.28) and (2.34) we have assumed that $\beta_{j} \leqslant 0$. In the case $\beta_{j}>0$, the quantum scalar field in the geometry of a single plate at $z=a_{j}$ has modes with $k_{p+1}=i / \beta_{j}$ for which the dependence on the coordinate $x^{p+1}$ has the form $e^{-z_{j} / \beta_{j}}$. In the case $1 / \beta_{j}>\omega_{0}$, for a part of these modes the energy is imaginary and the vacuum is unstable. In order to have a stable vacuum, in what follows, for non-Dirichlet boundary conditions, we shall assume that $1 / \beta_{j}<\omega_{0}$ and the mode with $k_{p+1}=i / \beta_{j}$ corresponds to a bound state. For $\beta_{j}>0$ and in the absence of purely imaginary roots of (2.17), in the right-hand side of the summation formula (2.25) the residue terms at $u= \pm i / b_{j}$ should be added (see [76]). Now the integrand in (2.33) has a simple pole at $k_{p+1}=$ $i s / \beta_{j}$ and after the rotation the contribution of the residue at that pole should be added. This contribution cancels the additional residue term in the right-hand side of (2.25). In the case when the equation (2.17) has purely imaginary roots the corresponding contributions have to be added to the modesum (2.21) for the Hadamard function. But the corresponding contributions should also be added in the left-hand side of
(2.25) and the further evaluation procedure remains the same. Hence, the expressions (2.28) and (2.34) are valid for all values of the coefficients in the Robin boundary conditions. The only restrictions come from the stability of the vacuum state: $1 / \beta_{j}<\omega_{0}$ and $y_{l}<\omega_{0}$. In the presence of compact dimensions with $\tilde{\alpha}_{l} \neq 0$ one has $\omega_{0}>m$ and these conditions are less restrictive than those in the case of trivial topology.

The current density in the boundary-free geometry is obtained by using the Hadamard function (2.31) and has been investigated in [53]. The corresponding charge density and the current densities along non-compact dimensions vanish. As can be seen from (2.28) and (2.34), the same holds in the case of the boundary-induced contributions in the VEVs. Hence, the only nonzero components correspond to the current density along compact dimensions.

\section{Vacuum currents in the geometry of a single plate}

In this section we investigate the VEV of the vacuum current density in the geometry of a single plate at $x^{p+1}=a_{j}$. This VEV is obtained with the help of the formula (2.6) by using the Hadamard function from (2.34). The component of the VEV of the current density along the $l$ th compact dimension is presented in decomposed form,

$\left\langle j^{l}\right\rangle_{j}=\left\langle j^{l}\right\rangle_{0}+\left\langle j^{l}\right\rangle_{j}^{(1)}$,

where $\left\langle j^{l}\right\rangle_{0}$ is the current density in the boundary-free geometry and $\left\langle j^{l}\right\rangle_{j}^{(1)}$ is the contribution induced by the presence of the plate.

The current density in the boundary-free geometry has been investigated in [53] and for completeness we will recall the main results. The current density is given by the formula

$$
\begin{aligned}
\left\langle j^{l}\right\rangle_{0}= & \frac{4 e L_{l} m^{D+1}}{(2 \pi)^{(D+1) / 2}} \sum_{n_{l}=1}^{\infty} n_{l} \sin \left(n_{l} \tilde{\alpha}_{l}\right) \\
& \times \sum_{\mathbf{n}_{q-1}} \cos \left(\mathbf{n}_{q-1} \cdot \tilde{\boldsymbol{\alpha}}_{q-1}\right) f_{\frac{D+1}{2}}\left(m g_{\mathbf{n}_{q}}\left(\mathbf{L}_{q}\right)\right),
\end{aligned}
$$

where $\tilde{\boldsymbol{\alpha}}_{q-1}=\left(\tilde{\alpha}_{p+2}, \ldots, \tilde{\alpha}_{l-1}, \tilde{\alpha}_{l+1}, \ldots, \tilde{\alpha}_{D}\right), \mathbf{n}_{q-1}=$ $\left(n_{p+2}, \ldots, n_{l-1}, n_{l+1}, \ldots, n_{D}\right)$, and $g_{\mathbf{n}_{q}}\left(\mathbf{L}_{q}\right)=\left(\sum_{i=p+2}^{D}\right.$ $\left.n_{i}^{2} L_{i}^{2}\right)^{1 / 2}$. The current density $\left\langle j^{l}\right\rangle_{0}$ is an odd periodic function of $\tilde{\alpha}_{l}$ with the period $2 \pi$ and an even periodic function of $\tilde{\alpha}_{r}, r \neq l$, with the same period. This corresponds to the periodicity in the magnetic flux with the period of flux quantum. An alternative expression for the current density in the boundary-free geometry is given by the formula

$$
\left\langle j^{l}\right\rangle_{0}=\frac{4 e L_{l} / V_{q}}{(2 \pi)^{(p+3) / 2}} \sum_{n=1}^{\infty} \frac{\sin \left(n \tilde{\alpha}_{l}\right)}{\left(n L_{l}\right)^{p+2}} \sum_{\mathbf{n}_{q-1}} g_{\frac{p+3}{2}}\left(n L_{l} \omega_{\mathbf{n}_{q-1}}\right),
$$


where we have defined the function

$g_{v}(x)=x^{v} K_{v}(x)$,

and

$\omega_{\mathbf{n}_{q-1}}^{2}=\omega_{\mathbf{n}_{q}}^{2}-k_{l}^{2}$.

In the model with a single compact dimension $(q=1)$ the representations (3.2) and (3.3) are identical.

When the length of the $l$ th compact dimension, $L_{l}$, is much larger than the other length scales, the behavior of the current density crucially depends whether the parameter

$\omega_{0 l}=\left(\sum_{i=p+2, \neq l}^{D} \tilde{\alpha}_{i}^{2} / L_{i}^{2}+m^{2}\right)^{1 / 2}$,

is zero or not. For $\omega_{0 l}=0$, which is realized for a massless field with $\tilde{\alpha}_{i}=0, i \neq l$, to the leading order we have

$\left\langle j^{l}\right\rangle_{0} \approx \frac{2 e \Gamma((p+3) / 2)}{\pi^{(p+3) / 2} L_{l}^{p+1} V_{q}} \sum_{n=1}^{\infty} \frac{\sin \left(n \tilde{\alpha}_{l}\right)}{n^{p+2}}$.

In this case, the leading term in the expansion of $V_{q}\left\langle j^{l}\right\rangle_{0} / L_{l}$ coincides with the current density in $(p+2)$-dimensional space with a single compact dimension of the length $L_{l}$. For $\omega_{0 l} \neq 0$ and for large values of $L_{l}$ one has

$\left\langle j^{l}\right\rangle_{0} \approx \frac{2 e V_{q}^{-1} \sin \left(\tilde{\alpha}_{l}\right) \omega_{0 l}^{p / 2+1}}{(2 \pi)^{p / 2+1} L_{l}^{p / 2}} e^{-L_{l} \omega_{0 l}}$,

and the current density is exponentially suppressed. In the opposite limit of small values for $L_{l}$, to the leading order we get

$\left\langle j^{l}\right\rangle_{0} \approx \frac{2 e \Gamma((D+1) / 2)}{\pi^{(D+1) / 2} L_{l}^{D}} \sum_{n=1}^{\infty} \frac{\sin \left(n \tilde{\alpha}_{l}\right)}{n^{D}}$.

The leading term does not depend on the mass and on the lengths of the other compact dimensions and coincides with the current density for a massless scalar field in the space with topology $R^{D-1} \times S^{1}$.

Now we turn to the investigation of the plate-induced contribution in the current density. By using the expression for the corresponding part in the Hadamard function from (2.34), we get the following expression:

$\left\langle j^{l}\right\rangle_{j}^{(1)}=\frac{e C_{p}}{2^{p} V_{q}} \sum_{\mathbf{n}_{q}} k_{l} \int_{\omega_{\mathbf{n}_{q}}}^{\infty} d y\left(y^{2}-\omega_{\mathbf{n}_{q}}^{2}\right)^{(p-1) / 2} e^{-2 y z_{j}} \frac{y \beta_{j}+1}{y \beta_{j}-1}$,

with the notations $z_{j}=\left|z-a_{j}\right|$ for the distance from the plate and

$C_{p}=\frac{\pi^{-(p+1) / 2}}{\Gamma((p+1) / 2)}$.
Recall that, in order to have a stable vacuum state with $\langle\varphi\rangle=0$, we have assumed that $1 / \beta_{j}<\omega_{0}$. Under this condition, the integrand in (3.10) is regular everywhere in the integration range. The integral in (3.10) is evaluated in the special cases of Dirichlet and Neumann boundary conditions with the result

$$
\left\langle j^{l}\right\rangle_{j}^{(1)}=\mp \frac{2 e / V_{q}}{(2 \pi)^{p / 2+1}} \sum_{\mathbf{n}_{q}} k_{l} \omega_{\mathbf{n}_{q}}^{p} f_{p / 2}\left(2 \omega_{\mathbf{n}_{q}} z_{j}\right),
$$

where the upper and lower signs correspond to Dirichlet and Neumann boundary conditions, respectively. Note that, in the problem with a fermionic field, obeying the bag boundary condition on the plate, the boundary-induced contribution vanishes for a massless field [55].

Let us consider the behavior of the plate-induced contribution in asymptotic regions of the parameters. At large distances from the plate, $z_{j} \gg L_{i}$, one has $z_{j} \omega_{\mathbf{n}_{q}} \gg 1$. Assuming that $\left|\tilde{\alpha}_{i}\right|<\pi$, the dominant contribution in (3.10) comes from the region near the lower limit of the integration and from the term with $n_{i}=0, i=p+2, \ldots, D$. To the leading order we find

$$
\left\langle j^{l}\right\rangle_{j}^{(1)} \approx \frac{e \tilde{\alpha}_{l} \omega_{0}^{(p-1) / 2} e^{-2 \omega_{0} z_{j}}}{(4 \pi)^{(p+1) / 2} V_{q} L_{l} z_{j}^{(p+1) / 2}} \frac{\omega_{0} \beta_{j}+1}{\omega_{0} \beta_{j}-1},
$$

and the current density is exponentially small. Note that the suppression is exponential for both massive and massless field.

For points close to the plate, $z_{j} \ll L_{i}$, in (3.10) the contribution of the terms with large values of $\left|n_{i}\right|$ dominates and this formula is not convenient for the asymptotic analysis and for numerical evaluations. In the case $\beta_{j} \leqslant 0$, an alternative expression is obtained by using the representation (A.6) for the Hadamard function. The first term in the right-hand side of this representation corresponds to the geometry with noncompactified $l$ th dimension and does not contribute to the current density along that direction. In the geometry of a single plate at $x^{p+1}=a_{j}$ the part in the Hadamard function induced by the compactification is given by the first term in the figure braces of (A.6). From this part, by making use of (2.6), for the VEV of the $l$ th component of the current density we get

$$
\begin{aligned}
\left\langle j^{l}\right\rangle_{j}= & \frac{2^{1-p / 2} e L_{l}}{\pi^{p / 2+2} V_{q}} \sum_{n=1}^{\infty} \frac{\sin \left(n \tilde{\alpha}_{l}\right)}{\left(n L_{l}\right)^{p+1}} \\
& \times \sum_{\mathbf{n}_{q-1}} \int_{0}^{\infty} d y g\left(z_{j}, y\right) g_{p / 2+1}\left(n L_{l} \sqrt{y^{2}+\omega_{\mathbf{n}_{q-1}}^{2}}\right),
\end{aligned}
$$


where we have defined the function

$$
\begin{aligned}
g\left(z_{j}, y\right) & =g_{j}(z, z, y)=1+\frac{1}{2} \sum_{s= \pm 1} e^{2 s i y z_{j}} \frac{i y \beta_{j}-s}{i y \beta_{j}+s} \\
& =1-\frac{\left(1-y^{2} \beta_{j}^{2}\right) \cos \left(2 y z_{j}\right)+2 y \beta_{j} \sin \left(2 y z_{j}\right)}{1+y^{2} \beta_{j}^{2}} .
\end{aligned}
$$

The part with the first term in the right-side of (3.15) corresponds to the current density in the boundary-free geometry. In this part the integration over $y$ is done with the help of the formula

$$
\int_{0}^{\infty} d y g_{\frac{p}{2}+1}\left(n L_{l} \sqrt{y^{2}+b^{2}}\right)=\sqrt{\pi / 2}\left(n L_{l}\right)^{-1} g_{\frac{p+3}{2}}\left(n L_{l} b\right),
$$

and one gets the expression (3.3).

Extracting the boundary-free part, for the plate-induced contribution from (3.14) we find

$$
\begin{aligned}
& \left\langle j^{l}\right\rangle_{j}^{(1)}=\frac{2^{-p / 2} e L_{l}}{\pi^{p / 2+2} V_{q}} \sum_{n=1}^{\infty} \frac{\sin \left(n \tilde{\alpha}_{l}\right)}{\left(n L_{l}\right)^{p+1}} \\
& \times \sum_{\mathbf{n}_{q-1}} \int_{0}^{\infty} d y g_{\frac{p}{2}+1}\left(n L_{l} \sqrt{y^{2}+\omega_{\mathbf{n}_{q-1}}^{2}}\right) \sum_{s= \pm 1} e^{2 s i y z j} \frac{i y \beta_{j}-s}{i y \beta_{j}+s} .
\end{aligned}
$$

In the case of single compact dimension one has $q=1, p=$ $D-2$, and the corresponding formula for the plate-induced contribution in the current density is obtained from (3.17) omitting the summation over $\mathbf{n}_{q-1}$ and putting $\omega_{\mathbf{n}_{q-1}}=m$.

An important issue in quantum field theory with boundaries is the appearance of surface divergences in the VEVs of local physical observables. Examples of the latter are the VEVs of the field squared and of the energy density. These divergences are a consequence of the oversimplification of a model where the physical interactions are replaced by the imposition of boundary conditions for all modes of a fluctuating quantum field. Of course, this is an idealization, as real physical systems cannot constrain all the modes (for a discussion of surface divergences and their physical interpretation see [1-6,78-94] and references therein). The appearance of divergences in the VEVs of physical quantities indicates that a more realistic physical model should be employed for their evaluation on the boundaries. An important feature, which directly follows from the representation (3.17), is that the VEV of the current density is finite on the plate. This is in sharp contrast with the behavior of the VEVs for the field squared and energy-momentum tensor. The finiteness of the current density on the boundary may be understood from general arguments. The divergences in local physical observables are determined by the local bulk and boundary geometries. If we consider the model with the topology $R^{p+2} \times T^{q-1}$ with the $l$ th dimension having the topology $R^{1}$, then in this model the $l$ th component of the current density vanishes by the symmetry. The compactification of the $l$ th dimension to $S^{1}$ does not change the two bulk end boundary local geometries and, hence, does not add new divergences to the VEVs compared with the model on $R^{p+2} \times T^{q-1}$.

In deriving (3.17) we have assumed that $\beta_{j} \leqslant 0$. In the case $\beta_{j}>0$ the contribution of the bound state should be added to (3.17). For $1 / \beta_{j}<\omega_{0 l}$, this contribution is obtained from the corresponding part in the Hadamard function, given by (A.7), and has the form

$$
\begin{aligned}
\left\langle j_{l}\right\rangle_{b j}^{(1)}= & -\frac{2^{2-p / 2} e L_{l} e^{-2 z_{j} / \beta_{j}}}{\pi^{p / 2+1} V_{q} \beta_{j}} \sum_{n=1}^{\infty} \frac{\sin \left(n \tilde{\alpha}_{l}\right)}{\left(n L_{l}\right)^{p+1}} \\
& \times \sum_{\mathbf{n}_{q-1}} g_{\frac{p}{2}+1}\left(n L_{l} \sqrt{\omega_{\mathbf{n}_{q-1}}^{2}-1 / \beta_{j}^{2}}\right) .
\end{aligned}
$$

In what follows for simplicity we shall consider the case $\beta_{j} \leqslant 0$. Recall that the representation (3.10) is valid for all values of $\beta_{j}$ from the range of the vacuum stability.

For Dirichlet and Neumann boundary conditions, after the evaluation of the integral in (3.17) by using the formula

$$
\begin{aligned}
& \int_{0}^{\infty} d y \cos \left(2 y z_{j}\right) g_{\frac{p}{2}+1}\left(n L_{l} \sqrt{y^{2}+b^{2}}\right) \\
& =\sqrt{\frac{\pi}{2}}\left(n L_{l}\right)^{p+2} \frac{g_{\frac{p+3}{2}}\left(b \sqrt{4 z_{j}^{2}+n^{2} L_{l}^{2}}\right)}{\left(4 z_{j}^{2}+n^{2} L_{l}^{2}\right)^{(p+3) / 2}},
\end{aligned}
$$

one gets

$$
\begin{aligned}
\left\langle j^{l}\right\rangle_{j}^{(1)}= & \mp \frac{4 e L_{l}^{2} / V_{q}}{(2 \pi)^{(p+3) / 2}} \sum_{n=1}^{\infty} \frac{n \sin \left(n \tilde{\alpha}_{l}\right)}{\left(4 z_{j}^{2}+n^{2} L_{l}^{2}\right)^{(p+3) / 2}} \\
& \times \sum_{\mathbf{n}_{q-1}} g_{\frac{p+3}{2}}\left(\omega_{\mathbf{n}_{q-1}} \sqrt{4 z_{j}^{2}+n^{2} L_{l}^{2}}\right),
\end{aligned}
$$

where the upper and lower signs correspond to Dirichlet and Neumann conditions, respectively. For a single compact dimension with the length $L$ and with the phase $\tilde{\alpha}$ in the periodicity condition for a massless field this gives

$$
\left\langle j^{l}\right\rangle_{j}^{(1)}=\mp \frac{2 \Gamma((D+1) / 2) e}{\pi^{(D+1) / 2} L^{D}} \sum_{n=1}^{\infty} \frac{n \sin (n \tilde{\alpha})}{\left(n^{2}+4 z_{j}^{2} / L^{2}\right)^{(D+1) / 2}} .
$$

Now, combining the expressions (3.3) and (3.20), we see that in the case of a Dirichlet boundary condition the boundaryfree and plate-induced parts of the current density cancel each other for $z_{j}=0$ and, hence, the total current vanishes on the plate. For a Neumann condition the current density on the plate is given by 


$$
\begin{aligned}
& \left\langle j^{l}\right\rangle_{j, z=a_{j}}=2\left\langle j^{l}\right\rangle_{0} \\
& \quad=\frac{8 e L_{l} / V_{q}}{(2 \pi)^{(p+3) / 2}} \sum_{n=1}^{\infty} \frac{\sin \left(n \tilde{\alpha}_{l}\right)}{\left(n L_{l}\right)^{p+2}} \sum_{\mathbf{n}_{q-1}} g_{\frac{p+3}{2}}\left(n L_{l} \omega_{\mathbf{n}_{q-1}}\right) .
\end{aligned}
$$

Note that the normal derivative of the current density on the plate vanishes for both Dirichlet and Neumann boundary conditions: $\left(\partial_{z}\left\langle j^{l}\right\rangle_{j}\right)_{z=a_{j}}=0$. This is not the case for a general Robin condition.

Let us consider the behavior of the plate-induced contribution in the current density in the limit $L_{i} \ll L_{l}$. In this investigation it is more convenient to use the representation (3.17). For $\sum_{i=p+2, \neq l}^{D} \tilde{\alpha}_{i}^{2} \neq 0$, the dominant contribution in the integral of (3.17) comes from the region near the lower limit of the integration and from the term $n=1, n_{i}=0$, $i=p+2, \ldots, D$, in the summation. The argument of the function $g_{p / 2+1}(x)$ in the integrand is large and we can use the asymptotic expression $g_{\nu}(x) \approx \sqrt{\pi / 2} x^{\nu-1 / 2} e^{-x}$. After some intermediate calculations, for the leading term we get

$$
\left\langle j^{l}\right\rangle_{j}^{(1)} \approx \frac{2 e\left(1-2 \delta_{0 \beta_{j}}\right)}{(2 \pi)^{p / 2+1} V_{q} L_{l}^{p / 2}} \frac{\omega_{0 l}^{p / 2+1} \sin \tilde{\alpha}_{l}}{e^{L_{l} \omega_{0 l}\left(1+2 z_{j}^{2} / L_{l}^{2}\right)}} .
$$

Here, we have additionally assumed that $L_{i} \ll\left|\beta_{j}\right|$ for $\beta_{j} \neq$ 0 . For $\tilde{\alpha}_{i}=0, i=p+2, \ldots, D, i \neq l$, the dominant contribution in (3.17) comes from the term $n_{i}=0, i=$ $p+2, \ldots, D$, with the leading term

$$
\begin{aligned}
& \frac{V_{q}}{L_{l}}\left\langle j^{l}\right\rangle_{j}^{(1)} \approx\left\langle j^{l}\right\rangle_{j, R^{p+1} \times S^{1}}^{(1)} \\
& =\frac{4 e}{(2 \pi)^{p / 2+2}} \sum_{n=1}^{\infty} \frac{\sin \left(n \tilde{\alpha}_{l}\right)}{\left(n L_{l}\right)^{p+1}} \int_{0}^{\infty} \mathrm{d} y \\
& \quad \times g_{\frac{p}{2}+1}\left(n L_{l} \sqrt{y^{2}+m^{2}}\right) \sum_{s= \pm 1} e^{2 s i y z_{j}} \frac{i y \beta_{j}-s}{i y \beta_{j}+s} .
\end{aligned}
$$

Here, $\left\langle j^{l}\right\rangle_{j, R^{p+1} \times S^{1}}^{(1)}$ is the plate-induced contribution in the current density for $(p+2)$-dimensional space with topology $R^{p+1} \times S^{1}$ (see (3.17) for the case $q=1$ and, hence, $\omega_{\mathbf{n}_{q-1}}=$ $m)$.

If the length of the $i$ th compact dimension is large, $i \neq l$, the dominant contribution to the sum over $n_{i}$ comes from large values of $\left|n_{i}\right|$ and in (3.17) we can replace the summation over $n_{i}$ by the integration in accordance with

$$
\sum_{n_{i}=-\infty}^{\infty} f\left(\left|k_{i}\right|\right) \rightarrow \frac{L_{i}}{\pi} \int_{0}^{\infty} \mathrm{d} x f(x) .
$$

The integral over $x$ is evaluated by using the formula (3.16). As a result, from (3.17), to the leading order, we obtain the current density along the $l$ th compact dimension for the spatial topology $R^{p+2} \times T^{q-1}$ with the lengths of the compact dimensions $\left(L_{p+2}, \ldots, L_{i-1}, L_{i+1}, \ldots, L_{D}\right)$.
Now let us consider the limiting case when $L_{l}$ is large compared with the other length scales in the problem, $L_{l} \gg$ $L_{i}, z_{j}, i \neq l$. The dominant contribution in (3.17) comes from the term $n_{i}=0, i \neq l$. For $\omega_{0 l} \neq 0$ we find

$$
\left\langle j^{l}\right\rangle_{j}^{(1)} \approx \frac{2 e\left(2 \delta_{\beta_{j}, \infty}-1\right)}{(2 \pi)^{p / 2+1} V_{q}} \frac{\sin \tilde{\alpha}_{l}}{L_{l}^{p / 2}} \omega_{0 l}^{p / 2+1} e^{-L_{l} \omega_{0 l}},
$$

where, for non-Neumann boundary conditions $\left(\beta_{j} \neq \infty\right)$, we have assumed that $\beta_{j} \omega_{0 l} \ll\left(L_{l} \omega_{0 l}\right)^{1 / 2}$. For $\omega_{0 l}=0$ the leading term is given by the expression

$$
\left\langle j^{l}\right\rangle_{j}^{(1)} \approx \frac{2 e\left(2 \delta_{\beta_{j}, \infty}-1\right)}{\pi^{(p+3) / 2} V_{q} L_{l}^{p+1}} \Gamma((p+3) / 2) \sum_{n=1}^{\infty} \frac{\sin \left(n \tilde{\alpha}_{l}\right)}{n^{p+2}} .
$$

Comparing with the corresponding asymptotics (3.7) and (3.8), we see that for non-Neumann boundary conditions, in the both cases $\omega_{0 l} \neq 0$ and $\omega_{0 l}=0$, the leading terms in the boundary-induced and boundary-free parts of the current density cancel each other.

An equivalent representation for the plate-induced current density is obtained from (3.17) rotating the integration contour in the complex plane $y$ by the angle $\pi / 2$ for the term with $s=1$ and by the angle $-\pi / 2$ for the term with $s=-1$. The integrals over the intervals $\left(0, \pm i \omega_{\mathbf{n}_{q-1}}\right)$ are canceled and we find

$$
\begin{aligned}
& \left\langle j^{l}\right\rangle_{j}^{(1)}=\frac{2^{-p / 2} e L_{l}}{\pi^{p / 2+1} V_{q}} \sum_{n=1}^{\infty} \frac{\sin \left(n \tilde{\alpha}_{l}\right)}{\left(n L_{l}\right)^{p+1}} \sum_{\mathbf{n}_{q-1}} \int_{\omega_{\mathbf{n}_{q-1}}}^{\infty} \mathrm{d} y \\
& \times e^{-2 y z_{j}} \frac{y \beta_{j}+1}{y \beta_{j}-1} w_{p / 2+1}\left(n L_{l} \sqrt{y^{2}-\omega_{\mathbf{n}_{q-1}}^{2}}\right),
\end{aligned}
$$

where

$w_{v}(x)=x^{v} J_{v}(x)$,

and $J_{v}(x)$ is the Bessel function. The equivalence of the representations (3.10) and (3.28) can also be directly seen by applying to the series over $n_{l}$ in (3.10) the relation

$$
\sum_{n_{l}=-\infty}^{+\infty} k_{l} g\left(\left|k_{l}\right|\right)=\frac{2 L_{l}}{\pi} \sum_{n=1}^{\infty} \sin \left(n \tilde{\alpha}_{l}\right) \int_{0}^{\infty} \mathrm{d} x x \sin \left(n L_{l} x\right) g(x) .
$$

The latter is a direct consequence of the Poisson resummation formula. After using (3.30) in (3.10), we introduce a new integration variable $u=\sqrt{y^{2}-x^{2}-\omega_{\mathbf{n}_{q-1}}^{2}}$ and then pass to polar coordinates in the $(u, x)$-plane. The integration over the polar angle is expressed in terms of the Bessel function and the representation (3.28) is obtained.

Another expression is obtained by applying to the series over $n_{l}$ in (3.10) the summation formula (A.1). For the series in (3.10) one has $g(u)=u$ and the first integral vanishes. 


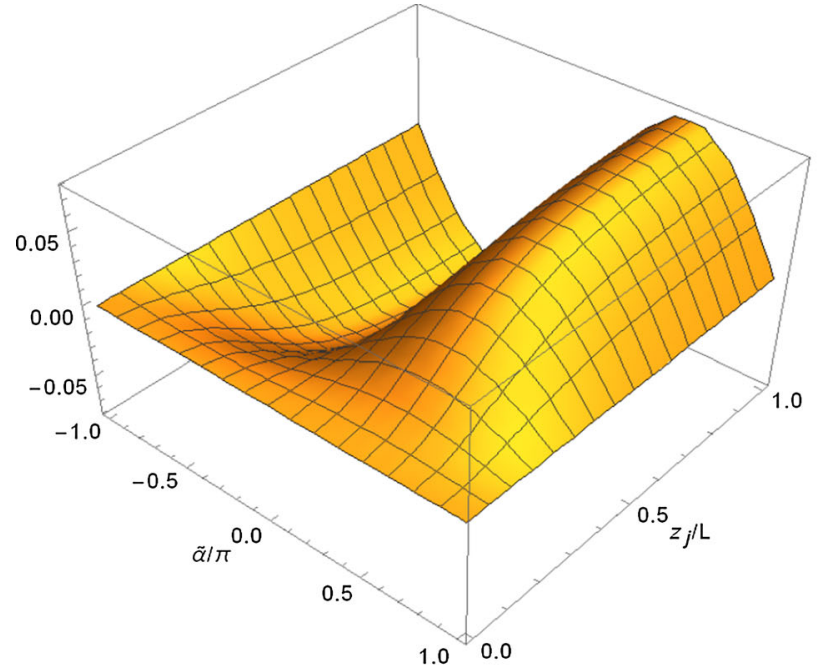

Fig. 1 The total current density, $L^{D}\left\langle j^{l}\right\rangle_{j} / e$, in the topology $R^{3} \times S^{1}$ for a $D=4$ massless scalar field with Dirichlet (left panel) and Neumann (right panel) boundary conditions in the geometry of a single plate, as

As a result, the plate-induced part in the VEV of the current density is presented as

$$
\begin{aligned}
& \left\langle j^{l}\right\rangle_{j}^{(1)}=-\frac{e C_{p} L_{l} \sin \tilde{\alpha}_{l}}{2^{p} \pi V_{q}} \sum_{\mathbf{n}_{q-1}} \int_{0}^{\infty} \mathrm{d} x \\
& \times \frac{x}{\cos h\left(L_{l} \sqrt{x^{2}+\omega_{\mathbf{n}_{q-1}}^{2}}\right)-\cos \tilde{\alpha}_{l}} \\
& \times \int_{0}^{x} \mathrm{~d} y \frac{\left(1-y^{2} \beta_{j}^{2}\right) \cos \left(2 y z_{j}\right)+2 y \beta_{j} \sin \left(2 y z_{j}\right)}{\left(1+y^{2} \beta_{j}^{2}\right)\left(x^{2}-y^{2}\right)^{(1-p) / 2}} .
\end{aligned}
$$

For Dirichlet and Neumann boundary conditions we obtain

$$
\begin{aligned}
& \left\langle j^{l}\right\rangle_{j}^{(1)}=\mp \frac{2 e L_{l} \sin \tilde{\alpha}_{l}}{(4 \pi)^{p / 2+1} V_{q} z_{j}^{p / 2}} \\
& \times \sum_{\mathbf{n}_{q-1}} \int_{0}^{\infty} \mathrm{d} x \frac{x^{p / 2+1} J_{p / 2}\left(2 x z_{j}\right)}{\cos h\left(L_{l} \sqrt{x^{2}+\omega_{\mathbf{n}_{q-1}}^{2}}\right)-\cos \tilde{\alpha}_{l}} .
\end{aligned}
$$

In Fig. 1, for the simplest Kaluza-Klein model with a single compact dimension of the length $L$ and with the phase $\tilde{\alpha}(D=4)$, we have plotted the total current density, $L^{D}\left\langle j^{l}\right\rangle_{j} / e$, for a massless scalar field in the geometry of a single plate as a function of the distance from the plate and of the phase $\tilde{\alpha}$. The left/right panel correspond to Dirichlet/Neumann boundary conditions. As has been already noticed before, in the Dirichlet case the total current density vanishes on the plate.

For the same model, Fig. 2 presents the plate-induced contribution to the current density as a function of the distance from the plate for various values of the coefficients in the

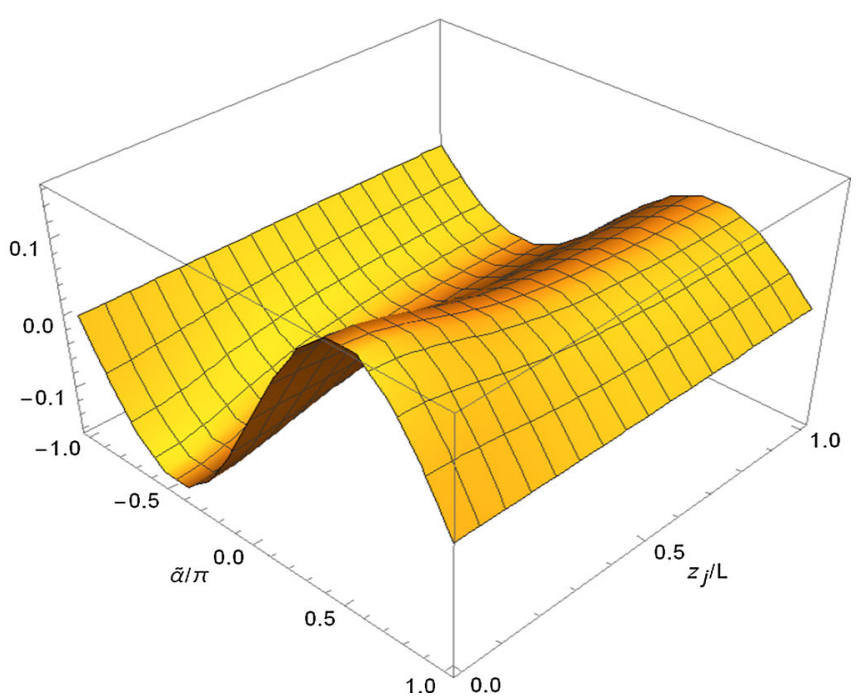

a function of the phase in the quasiperiodicity boundary condition and of the distance from the plate

Robin boundary condition (left panel) and as a function of the ratio $\beta_{j} / L$ (right panel). The numbers near the curves on the right panel correspond to the value of $\beta_{j} / L$. The left panel is plotted for the fixed value of the relative distance from the plate $z_{j} / L=0.3$. On both panels, the dashed curves are plotted for Dirichlet and Neumann boundary conditions. For the phase in the quasiperiodicity condition we have taken $\tilde{\alpha}=\pi / 2$. On the right panel, for the values of $\beta_{j} / L$ between the ordinate axis and the vertical dotted line $\left(\beta_{j} / L=1 / \tilde{\alpha}\right)$ the vacuum is unstable.

\section{Current density between two plates}

Now we turn to the geometry of two plates. In the region $a_{1} \leqslant$ $x^{p+1} \leqslant a_{2}$, by using the formula (2.28) for the Hadamard function, the VEV of the current density is decomposed as

$$
\begin{gathered}
\left\langle j^{l}\right\rangle=\left\langle j^{l}\right\rangle_{j}+\frac{e C_{p}}{2^{p-1} V_{q}} \sum_{\mathbf{n}_{q}} k_{l} \int_{\omega_{\mathbf{n}_{q}}}^{\infty} \mathrm{d} y \\
\times \frac{\left(y^{2}-\omega_{\mathbf{n}_{q}}^{2}\right)^{(p-1) / 2} g\left(z_{j}, i y\right)}{c_{1}(\text { ay }) c_{2}(\text { ay }) e^{2 a y}-1} .
\end{gathered}
$$

Here, the second term in the right-hand side is induced by the plate at $x^{p+1}=a_{j^{\prime}}, j^{\prime} \neq j$.

Extracting from the second term in the right-hand side of (4.1) the part induced by the second plate when the first one is absent, the current density is written in a more symmetric form:

$$
\left\langle j^{l}\right\rangle=\left\langle j^{l}\right\rangle_{0}+\sum_{j=1,2}\left\langle j^{l}\right\rangle_{j}^{(1)}+\Delta\left\langle j^{l}\right\rangle
$$




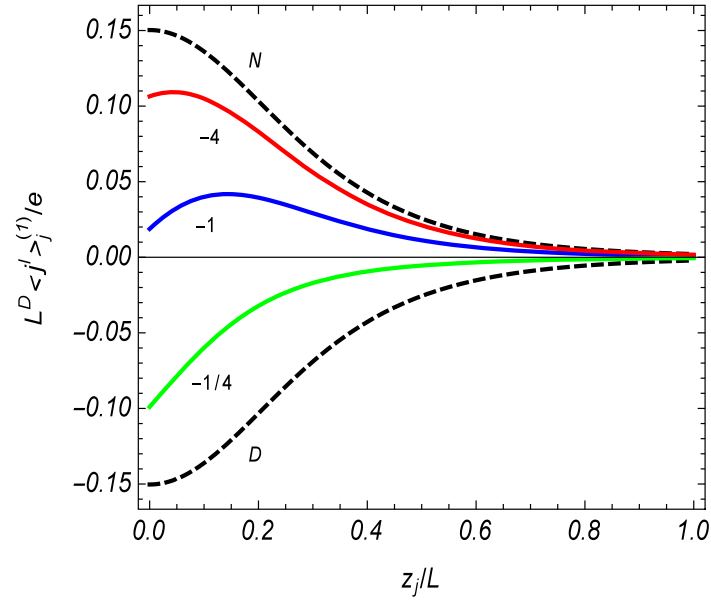

Fig. 2 The plate-induced contribution to the current density for the model corresponding to Fig. 1 as a function of the distance from the plate (left panel) for different values of the ratio $\beta_{j} / L$ (numbers near

where the interference part is given by the expression

$$
\begin{aligned}
\Delta\left\langle j^{l}\right\rangle= & \frac{e C_{p}}{2^{p} V_{q}} \sum_{\mathbf{n}_{q}} k_{l} \int_{\omega_{\mathbf{n}_{q}}}^{\infty} \mathrm{d} y\left(y^{2}-\omega_{\mathbf{n}_{q}}^{2}\right)^{\frac{p-1}{2}} \\
& \times \frac{2+\sum_{j=1,2} e^{-2 y z_{j}} / c_{j}(\text { ay })}{c_{1}(\text { ay }) c_{2}(\text { ay }) e^{2 a y}-1} .
\end{aligned}
$$

By taking into account the expression for the current density in the geometry of a single plate, for the total current density we can also write

$$
\begin{aligned}
\left\langle j^{l}\right\rangle= & \left\langle j^{l}\right\rangle_{0}+\frac{e C_{p}}{2^{p} V_{q}} \sum_{\mathbf{n}_{q}} k_{l} \int_{\omega_{\mathbf{n}_{q}}}^{\infty} \mathrm{d} y\left(y^{2}-\omega_{\mathbf{n}_{q}}^{2}\right)^{\frac{p-1}{2}} \\
& \times \frac{2+\sum_{j=1,2} c_{j}(\text { ay }) e^{2 y z_{j}}}{c_{1}(\text { ay }) c_{2}(\text { ay }) e^{2 a y}-1} .
\end{aligned}
$$

For special cases of Dirichlet and Neumann boundary conditions on both plates the general formula is simplified to

$$
\begin{aligned}
\left\langle j^{l}\right\rangle= & \left\langle j^{l}\right\rangle_{0}+\frac{e C_{p}}{2^{p} V_{q}} \sum_{\mathbf{n}_{q}} k_{l} \int_{\omega_{\mathbf{n}_{q}}}^{\infty} \mathrm{d} y\left(y^{2}-\omega_{\mathbf{n}_{q}}^{2}\right)^{\frac{p-1}{2}} \\
& \times \frac{2 \mp \sum_{j=1,2} e^{2 y z_{j}}}{e^{2 a y}-1}
\end{aligned}
$$

where, as before, the upper and lower signs correspond to Dirichlet and Neumann boundary conditions, respectively. In particular, for Dirichlet boundary condition the part induced by the second plate vanishes on the first plate. Note that in the system of two fields with Dirichlet and Neumann conditions the distribution of the total current density in the region between the plates is uniform and the current density vanishes in the regions $z<a_{1}$ and $z>a_{2}$. Another form for (4.5) is obtained by making use of the expansion

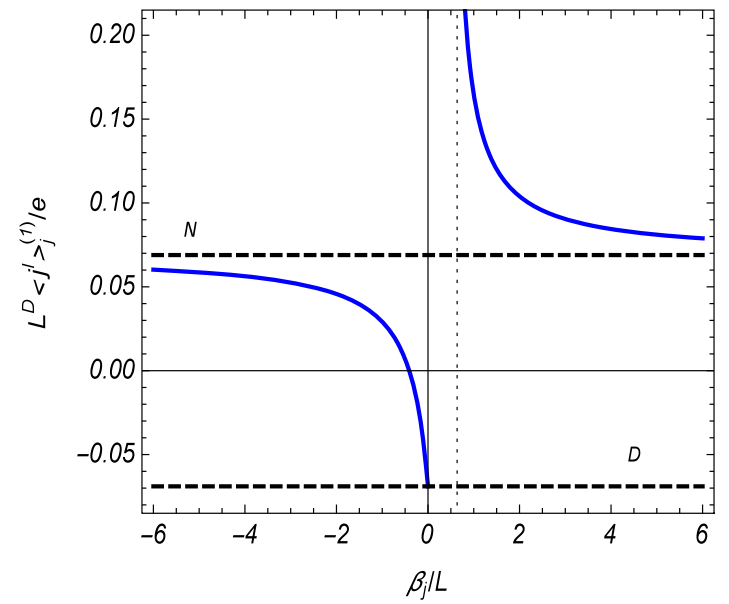

the curves) and as a function of $\beta_{j} / L$ (right panel) for $z_{j} / L=0.3$. The dashed curves correspond to Dirichlet and Neumann boundary conditions and the graphs are plotted for $\tilde{\alpha}=\pi / 2$

$\frac{1}{e^{2 a y}-1}=\sum_{n=1}^{\infty} e^{-2 n a y}$

After the integration over $y$ we get

$$
\begin{aligned}
\left\langle j^{l}\right\rangle= & \left\langle j^{l}\right\rangle_{0}+\frac{2 e / V_{q}}{(2 \pi)^{p / 2+1}} \sum_{n=1}^{\infty} \sum_{\mathbf{n}_{q}} k_{l} \omega_{\mathbf{n}_{q}}^{p}\left[2 f_{\frac{p}{2}}\left(2 n a \omega_{\mathbf{n}_{q}}\right)\right. \\
& \left.\mp \sum_{j=1,2} f_{\frac{p}{2}}\left(2\left(n a-z_{j}\right) \omega_{\mathbf{n}_{q}}\right)\right] .
\end{aligned}
$$

A similar representation for the interference part $\Delta\left\langle j^{l}\right\rangle$ is obtained from (4.7) by the replacement $z_{j} \rightarrow-z_{j}$. For Dirichlet boundary condition, on the plates, $z=a_{j}$, one has

$\Delta\left\langle j^{l}\right\rangle_{z=a_{j}}=\frac{2 e / V_{q}}{(2 \pi)^{p / 2+1}} \sum_{\mathbf{n}_{q}} k_{l} \omega_{\mathbf{n}_{q}}^{p} f_{\frac{p}{2}}\left(2 a \omega_{\mathbf{n}_{q}}\right)$.

Combining this result with the formulas for single plates, we see that in the case of Dirichlet boundary condition the total current vanishes on the plates: $\left\langle j^{l}\right\rangle_{z=a_{j}}=0$.

An equivalent representation for the current density in the region between the plates and for Robin conditions is obtained by using the representation (A.6) for the corresponding Hadamard function:

$$
\begin{aligned}
\left\langle j^{l}\right\rangle= & \left\langle j^{l}\right\rangle_{j}+\frac{2^{1-p / 2} e L_{l}}{\pi^{p / 2+1} V_{q}} \sum_{n=1}^{\infty} \frac{\sin \left(n \tilde{\alpha}_{l}\right)}{\left(n L_{l}\right)^{p+1}} \sum_{\mathbf{n}_{q-1}} \int_{\omega_{\mathbf{n}_{q-1}}}^{\infty} \mathrm{d} y \\
& \times \frac{w_{p / 2+1}\left(n L_{l} \sqrt{y^{2}-\omega_{\mathbf{n}_{q-1}}^{2}}\right)}{c_{1}(a y) c_{2}(a y) e^{2 a y}-1} g\left(z_{j}, i y\right) .
\end{aligned}
$$


Combining Eqs. (3.28) and (4.9), for the total current density we find

$$
\begin{aligned}
& \left\langle j^{l}\right\rangle=\left\langle j^{l}\right\rangle_{0}+\frac{2^{-p / 2} e L_{l}}{\pi^{p / 2+1} V_{q}} \sum_{n=1}^{\infty} \frac{\sin \left(n \tilde{\alpha}_{l}\right)}{\left(n L_{l}\right)^{p+1}} \sum_{\mathbf{n}_{q-1}} \int_{\omega_{\mathbf{n}_{q-1}}}^{\infty} \mathrm{d} y \\
& \times \frac{2+\sum_{j=1,2} e^{2 y z_{j}} c_{j}(\text { ay })}{c_{1}(\text { ay }) c_{2}(\text { ay }) e^{2 a y}-1} w_{p / 2+1}\left(n L_{l} \sqrt{y^{2}-\omega_{\mathbf{n}_{q-1}}^{2}}\right) .
\end{aligned}
$$

Now, by taking into account the expression (3.28) for the single-plate-induced part, from (4.9) for the interference part we get

$$
\begin{aligned}
& \Delta\left\langle j^{l}\right\rangle=\frac{2^{-p / 2} e L_{l}}{\pi^{p / 2+1} V_{q}} \sum_{n=1}^{\infty} \frac{\sin \left(n \tilde{\alpha}_{l}\right)}{\left(n L_{l}\right)^{p+1}} \sum_{\mathbf{n}_{q-1}} \int_{\omega_{\mathbf{n}_{q-1}}}^{\infty} \mathrm{d} y \\
& \times \frac{2+\sum_{j=1,2} e^{-2 y z_{j}} / c_{j}(\text { ay })}{c_{1}\left(\text { ay) } c_{2}(\text { ay }) e^{2 a y}-1\right.} w_{p / 2+1}\left(n L_{l} \sqrt{y^{2}-\omega_{\mathbf{n}_{q-1}}^{2}}\right) .
\end{aligned}
$$

The equivalence of the representations (4.4) and (4.9) can be seen directly by using the formula (3.30) in a way similar to that for the geometry of a single plate.

For Dirichlet and Neumann conditions, after using the expansion (4.6), the integral over $y$ in (4.10) is expressed in terms of the MacDonald function and one gets the representation

$$
\begin{aligned}
\left\langle j^{l}\right\rangle= & \frac{2^{(1-p) / 2} e L_{l}^{2}}{\pi^{(p+3) / 2} V_{q}} \sum_{n=1}^{\infty} n \sin \left(n \tilde{\alpha}_{l}\right) \sum_{\mathbf{n}_{q-1}} \omega_{\mathbf{n}_{q-1}}^{p+3} \\
& \times \sum_{r=-\infty}^{\infty}\left\{f_{\frac{p+3}{2}}\left(\omega_{\mathbf{n}_{q-1}} \sqrt{4(r a)^{2}+n^{2} L_{l}^{2}}\right)\right. \\
& \left.\mp f_{\frac{p+3}{2}}\left(\omega_{\mathbf{n}_{q-1}} \sqrt{4\left(r a-z+a_{1}\right)^{2}+n^{2} L_{l}^{2}}\right)\right\},
\end{aligned}
$$

where we have taken into account the expression (3.3) for the current density in the boundary-free geometry. In the model with a single compact dimension with the length $L$ and for a massless field, from (4.12) we find

$$
\begin{aligned}
\left\langle j^{l}\right\rangle= & \frac{2 \Gamma((D+1) / 2) e}{\pi^{(D+1) / 2} L^{D}} \sum_{n=1}^{\infty} \sum_{r=-\infty}^{\infty} n \sin (n \tilde{\alpha}) \\
& \times\left\{\left[4(r a / L)^{2}+n^{2}\right]^{-\frac{D+1}{2}}\right. \\
& \left.\mp\left[4\left(r a-z+a_{1}\right)^{2} / L^{2}+n^{2}\right]^{-\frac{D+1}{2}}\right\} .
\end{aligned}
$$

In the case of Dirichlet boundary condition on the left plate, $x^{p+1}=a_{1}$, and Neumann boundary condition on the right one, $x^{p+1}=a_{2}$, the corresponding formulas are obtained from (4.12) and (4.13) with the upper sign, adding the factor $(-1)^{r}$ in the summation over $r$. The corresponding current density vanishes on the left plate. From (4.12) we can also see that the normal derivative of the current density vanishes on the plates for both Dirichlet and Neumann boundary conditions.

In the limit $a \ll L_{i}, i \neq l$, the dominant contribution to the series over $\mathbf{n}_{q-1}$ in (4.11) comes from large values of $\left|n_{i}\right|$, $i \neq l$, and we can replace the summation by the integration in accordance with

$$
\sum_{\mathbf{n}_{q-1}} f\left(\omega_{\mathbf{n}_{q-1}}\right) \rightarrow \frac{2(4 \pi)^{(1-q) / 2} V_{q}}{\Gamma((q-1) / 2) L_{l}} \int_{0}^{\infty} d u u^{q-2} f\left(\sqrt{u^{2}+m^{2}}\right) .
$$

Changing the integration variable $y$ to $x=\sqrt{y^{2}-u^{2}}$, we introduce polar coordinates in the $(u, x)$-plane. After the integration over the polar angle, we get

$\Delta\left\langle j^{l}\right\rangle \approx \Delta\left\langle j^{l}\right\rangle_{R^{D} \times S^{1}}$,

where $\Delta\left\langle j^{l}\right\rangle_{R^{D} \times S^{1}}$ is the corresponding quantity in the geometry of a single compact dimension with the length $L_{l}$. The expression for $\Delta\left\langle j^{l}\right\rangle_{R^{D} \times S^{1}}$ is obtained from (4.11) taking $p=D-2, V_{q}=L_{l}, \omega_{\mathbf{n}_{q-1}}=m$, and omitting the summation over $\mathbf{n}_{q-1}$. If, in addition, $a m \ll 1$, one finds

$$
\begin{aligned}
\Delta\left\langle j^{l}\right\rangle & \approx \frac{2 e}{(2 \pi)^{D / 2} a} \sum_{n=1}^{\infty} \frac{\sin \left(n \tilde{\alpha}_{l}\right)}{\left(n L_{l}\right)^{D-1}} \int_{0}^{\infty} \mathrm{d} y \\
& \times \frac{2+\sum_{j=1,2} e^{-2 y z_{j} / a} / c_{j}(y)}{c_{1}(y) c_{2}(y) e^{2 y}-1} w_{D / 2}\left(n L_{l} y / a\right) .
\end{aligned}
$$

Now let us also assume that $a \ll L_{i}, m^{-1}$, for all $i=$ $p+2, \ldots, D$. This means that the separation between the plates is smaller than all other length scales in the problem. In order to estimate the integral in (4.16), we note that for a fixed $b$ and for $\lambda \rightarrow+\infty$, the dominant contribution to the integral $\int_{0}^{\infty} \mathrm{d} y f(y) e^{-b y} w_{D / 2}(\lambda y)$ comes from the region with $y \lesssim a / L$. By taking into account that

$$
\int_{0}^{\infty} \mathrm{d} y e^{-b y} w_{D / 2}(\lambda y)=\frac{2^{D / 2} \lambda^{D} \Gamma((D+1) / 2)}{\sqrt{\pi}\left(b^{2}+\lambda^{2}\right)^{(D+1) / 2}},
$$

to the leading order we get

$$
\int_{0}^{\infty} \mathrm{d} y f(y) e^{-b y} w_{D / 2}(\lambda y) \approx \frac{2^{D / 2}}{\sqrt{\pi} \lambda} \Gamma((D+1) / 2) f(0) .
$$

For the integral in (4.16) we take $b=2$ and

$$
f(y)=\frac{2+\sum_{j=1,2} e^{-2 y z_{j} / a} / c_{j}(y)}{c_{1}(y) c_{2}(y)-e^{-2 y}} .
$$

In the case of non-Neumann boundary conditions one has $f(0)=1$ and, hence,

$\Delta\left\langle j^{l}\right\rangle \approx \frac{2 e \Gamma((D+1) / 2)}{\pi^{(D+1) / 2} L^{D}} \sum_{n=1}^{\infty} \frac{\sin \left(n \tilde{\alpha}_{l}\right)}{n^{D}}$. 


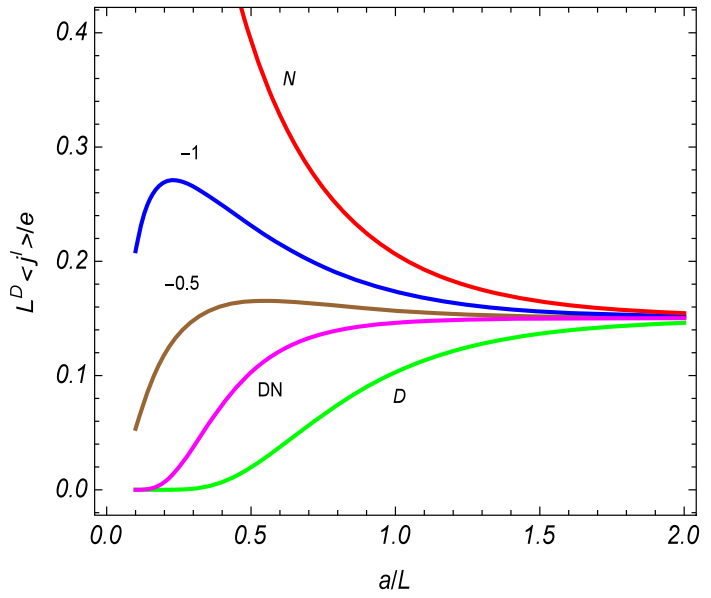

Fig. 3 The VEV of the current density in the region between the plates evaluated at $z=a / 2$, as a function of the separation between the plates. The graphs are plotted for Dirichlet and Neumann boundary conditions on both plates, for Dirichlet condition on the left plate and Neumann condition on the right one, and for Robin boundary conditions with the values of $\beta_{j} / L$ given near the curves. For the phase we have taken the value $\tilde{\alpha}=\pi / 2$

Combining this result with the expressions from the previous section for the geometry of a single plate, we conclude that $\lim _{a \rightarrow 0}\left\langle j^{l}\right\rangle=0$, i.e., for non-Neumann boundary conditions the total current density in the region between the plates tends to zero for small separations between the plates. For nonNeumann boundary condition on one plate and Neumann boundary condition on the other we have $f(0)=-1$ and the corresponding formula is obtained from (4.20) changing the sign of the right-hand side. In this case we have again $\lim _{a \rightarrow 0}\left\langle j^{l}\right\rangle=0$.

For Neumann boundary condition on both plates, for the function in (4.19) we have $f(y) \sim 2 / y, y \rightarrow 0$. In order

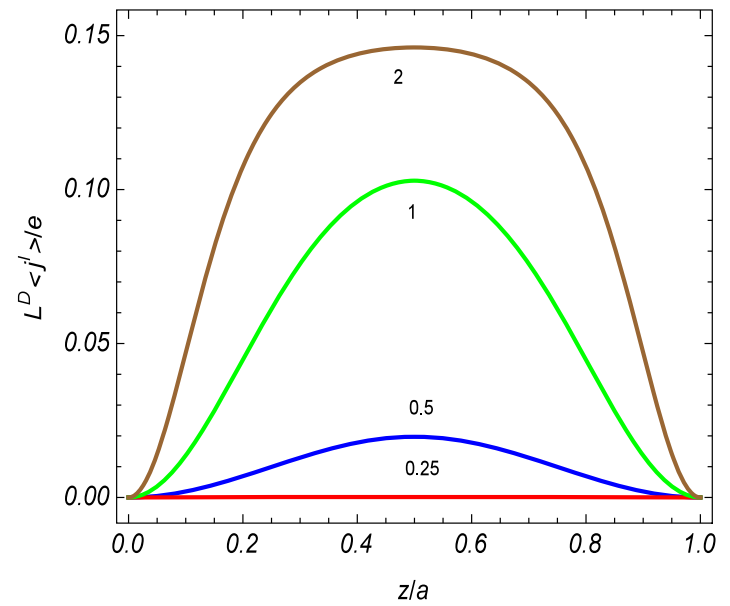

Fig. 4 The current density between the plates as a function of the relative distance from the left plate in the model with a single compact dimension. The graphs are plotted for a massless field with the parame- to obtain the leading term in the asymptotic expansion for small values of $a$ it is more convenient to use the expression (4.13) with the lower sign instead of the right-hand side of (4.16). For small $a / L$ the dominant contribution in (4.13) comes from large values of $r$ and, to the leading order, we replace the corresponding summation by the integration. For the leading term this gives

$\left\langle j^{l}\right\rangle \approx \frac{2 e \Gamma(D / 2)}{\pi^{D / 2} L^{D-1} a} \sum_{n=1}^{\infty} \frac{\sin (n \tilde{\alpha})}{n^{D-1}}$,

and for Neumann boundary condition the current density diverges in the limit $a \rightarrow 0$ like $1 / a$. The described features in the behavior of the vacuum current density, $L^{D}\left\langle j^{l}\right\rangle / e$, in the region between the plates located at $z=0$ and $z=a$, as a function of the separation between the plates, is illustrated in Fig. 3 for a $D=4$ massless scalar field in the model with a single compact dimension of the length $L$ and of the phase $\tilde{\alpha}$. The graphs are plotted for $z=a / 2$ and $\tilde{\alpha}=\pi / 2$, in the cases of Dirichlet (D), Neumann (N) boundary conditions on both plates, for Dirichlet boundary condition at $z=0$ and Neumann boundary condition at $z=a(\mathrm{DN})$, and for Robin boundary conditions with $\beta_{j} / L=-0.5$ and $\beta_{j} / L=-1$ (numbers near the curves). At large separations between the plates, the boundary-induced effects are small and the current density coincides with that in the boundary-free geometry.

In Fig. 4, in the model with a single compact dimension of the length $L$ and for a $D=4$ massless scalar field with Dirichlet (left panel) and Neumann (right panel) boundary conditions, we have plotted the total current density as a function of the ratio $z / a$ in the region between the plates. The numbers near the curves correspond to the values of $a / L$ and the graphs are plotted for $\tilde{\alpha}=\pi / 2$. The features, obtained before on the base of asymptotic analysis, are

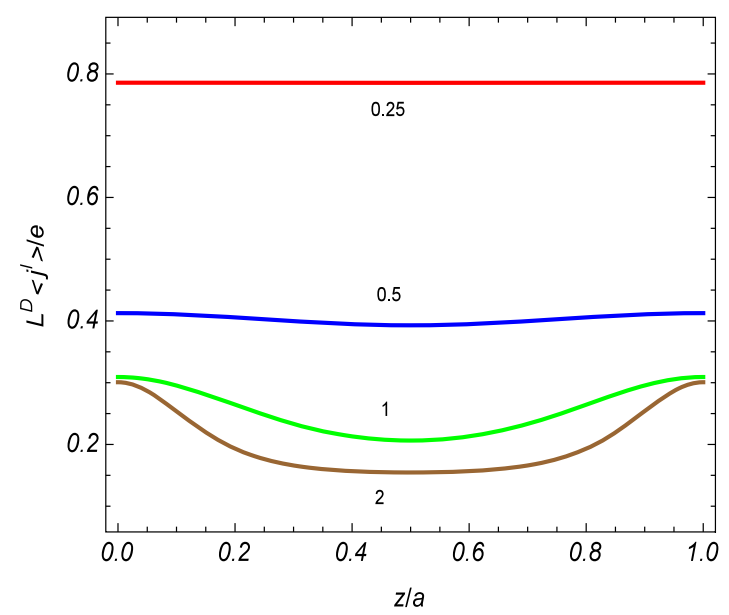

ter $\tilde{\alpha}=\pi / 2$ and with Dirichlet (left panel) and Neumann (right panel) boundary conditions. The numbers near the curves correspond to the values of $a / L$ 

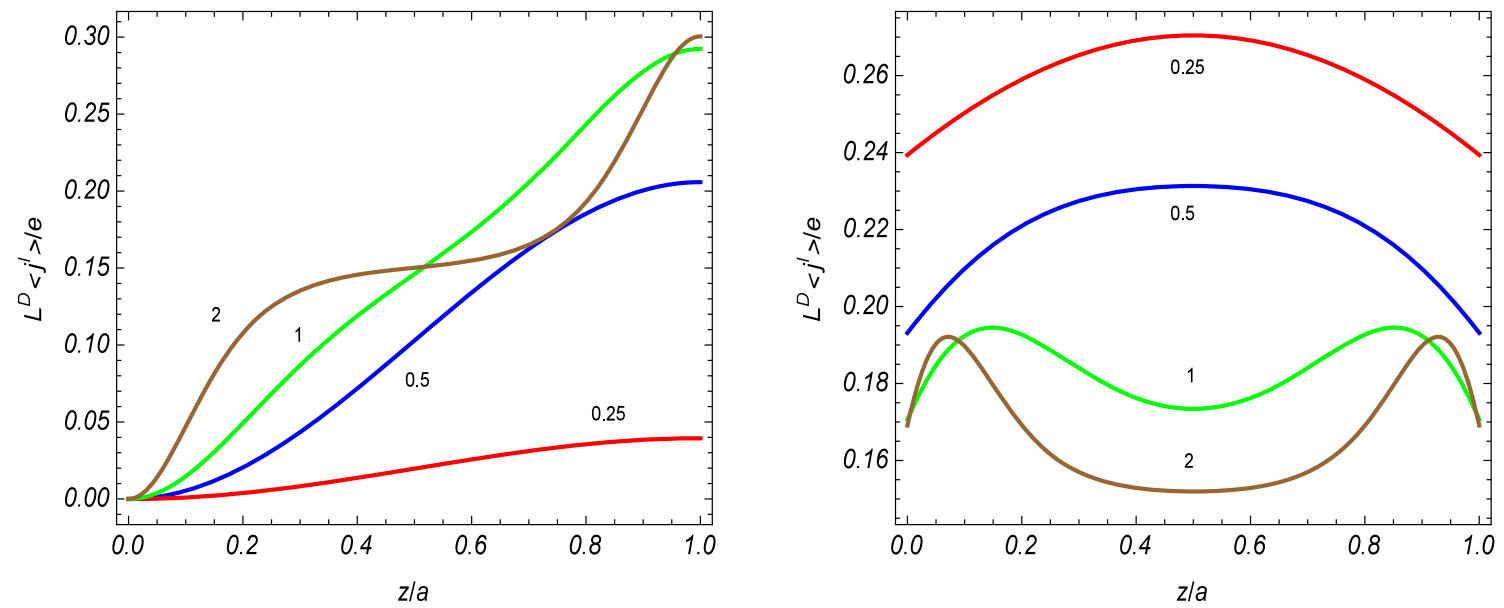

Fig. 5 The same as in Fig. 4 for Dirichlet boundary condition on the left plate and Neumann condition on the right one (left panel). The right panel is plotted for Robin boundary condition on both plates with $\beta_{1} / L=\beta_{2} / L=-1$

clearly seen from the graphs: the current density for Dirichlet/Neumann scalar decreases/increases with decreasing separation between the plates and for Dirichlet scalar it vanishes on the plates.

The same graphs for Dirichlet boundary condition on the left plate and Neumann condition on the right one are presented on the left panel of Fig. 5. The right panel in Fig. 5 is plotted for Robin boundary condition on both plates with $\beta_{1} / L=\beta_{2} / L=-1$. In the Robin case, the current density decreases with the further decrease of the separation between the plates and it tends to zero in the limit $a \rightarrow 0$, in accordance with the general analysis described above.

\section{Conclusion}

In the present paper we have investigated the influence of parallel flat boundaries on the VEV of the current density for a charged scalar field in a flat spacetime with toroidally compactified spatial dimensions, assuming the presence of a constant gauge field. The effect of the latter on the current is similar to the Aharonov-Bohm effect and is caused by the nontrivial topology of the background space. Along compact dimensions we have considered quasiperiodicity conditions with general phases. The special cases of twisted and untwisted fields are the configurations most frequently discussed in the literature. By a gauge transformation, the problem with a constant gauge field is mapped to the one with zero field, shifting the phases in the periodicity conditions by an amount proportional to the magnetic flux enclosed by a compact dimension in the initial representation of the model. On the plates we employed Robin boundary conditions, in general, with different coefficients on the left and right plates. The Robin boundary conditions for bulk fields naturally arise in braneworld scenario and the boundaries considered here may serve as a simple model for the branes.
We considered a free field theory and all the information on the properties of the vacuum state is encoded in two-point functions. Here we chose the Hadamard function. The VEV of the current density is obtained from this function in the coincidence limit by using (2.6). For the evaluation of the Hadamard function we have employed a direct summation over the complete set of modes. In the region between the plates the eigenvalues of the momentum component perpendicular to the plates are quantized by the boundary conditions on the plates and are given implicitly, in terms of solutions of the transcendental equation (2.17). Depending on the values of the Robin coefficients, this equation may have purely imaginary solutions $y= \pm i y_{l}$. In order to have a stable vacuum with $\langle\varphi\rangle=0$, we assume that $\omega_{0}>y_{l}$. Compared to the case of the bulk with trivial topology, this constraint in models with compact dimensions is less restrictive. The eigenvalues of the momentum components along compact dimensions are quantized by the periodicity conditions and are determined by (2.12). The application of the generalized Abel-Plana formula for the summation over the roots of (2.17) allowed us to extract from the Hadamard function the part corresponding to the geometry with a single plate and to present the second-plate-induced contribution in the form which does not require the explicit knowledge of the eigenmodes for $k_{p+1}$ ' [see (2.28)]. In addition, the corresponding integrand decays exponentially in the upper limit. A similar representation, (2.34), is obtained for the Hadamard function in the geometry of a single plate. The second term in the right-hand side of this representation is the boundary-induced contribution. An alternative representation for the Hadamard function, (A.6), is obtained in the "Appendix", by making use of the summation formula (A.1). The second term in the right-hand side of this representation is the contribution induced by the compactification of the $l$ th dimension. 
The VEVs of the charge density and the components of the current density along non-compact dimensions vanish. The current density along compact dimensions is a periodic function of the magnetic flux with the period equal to the flux quantum. The component along the $l$ th compact dimension is an odd function of the phase $\tilde{\alpha}_{l}$ and an even function of the remaining phases $\tilde{\alpha}_{i}, i \neq l$. First we have considered the geometry with a single plate. The VEV of the current density is decomposed into the boundary-free and plate-induced parts. The boundary-free contribution was investigated in [53] and we have been mainly concerned with the plate-induced part, given by (3.10). For special cases of Dirichlet and Neumann boundary conditions the corresponding expression is simplified to (3.12). The plate-induced part has opposite signs for Dirichlet and Neumann conditions. At distances from the plate larger than the lengths of compact dimensions the asymptotic is described by (3.13) and the plate-induced contribution is exponentially small. For the investigation of the near-plate asymptotic of the current density it is more convenient to use the representation (3.17) for the general Robin case and (3.20) for Dirichlet and Neumann conditions. From these representations it follows that the current density is finite on the plate. This property is in sharp contrast with the behavior of the VEVs of the field squared and of the energy-momentum tensor which diverge on the plate. For Dirichlet boundary condition the current density vanishes on the plate and for Neumann condition its value on the plate is two times larger than the current density in the boundary-free geometry. The normal derivative of the current density vanishes on the plate for both Dirichlet and Neumann conditions. This is not the case for general Robin condition. The behavior of the plate-induced part of the current density along $l$ th dimension, in the limit when the lengths of the other compact dimensions are much smaller than $L_{l}$, crucially depend on whether the phases $\tilde{\alpha}_{i}, i \neq l$, are zero or not. For $\sum_{i \neq l} \tilde{\alpha}_{i}^{2} \neq 0$ one has $\omega_{0 l} \neq 0$ and the corresponding asymptotic expression is given by (3.23). In this case the plate-induced contribution is exponentially suppressed. For $\tilde{\alpha}_{i}=0, i \neq l$, the leading term in the asymptotic expansion, multiplied by $V_{q} / L_{l}$, coincides with the corresponding current density for $(p+2)$-dimensional space with topology $R^{p+1} \times S^{1}$. In the limit when the length of the $l$ th dimension is much larger than the other length scales of the model, the behavior of the plate-induced contribution to the current density is essentially different for the cases $\omega_{0 l} \neq 0$ and $\omega_{0 l}=0$. In the former case the leading term is given by (3.26) and the current density is suppressed by the factor $e^{-L_{l} \omega_{0 l}}$. In the second case, for the leading term one has Eq. (3.27) and its behavior, as a function of $L_{l}$, is a power law. In both cases and for non-Neumann boundary conditions, the leading terms in the boundary-induced and boundary-free parts of the current density cancel each other.
For the current density in the region between the plates we have provided various decompositions ((4.1), (4.2), (4.4) for general Robin boundary conditions and (4.5), (4.7), (4.12) for special cases of Dirichlet and Neumann conditions). In the case of Dirichlet boundary condition the total current vanishes on the plates. The normal derivative vanishes on the plates for both Dirichlet and Neumann cases. In the limit when the separation between the plates is smaller than all the length scales in the problem, the behavior of the current density is essentially different for non-Neumann and Neumann boundary conditions. In the former case, the total current density in the region between the plates tends to zero. For Neumann boundary condition on both plates, for small separations the total current density is dominated by the interference part and it diverges inversely proportional to the separation [see (4.21)]. The results of the present paper may be applied to Kaluza-Klein-type models in the presence of branes (for $D>3$ ) and to planar condensed matter systems (for $D=2$ ), described within the framework of an effective field theory. In particular, in the former case, the vacuum currents along compact dimensions generate magnetic fields in the non-compactified subspace. The boundaries discussed above can serve as a simple model for the edges of planar systems.

Acknowledgments N. A. S. was supported by the State Committee of Science Ministry of Education and Science RA, within the frame of Research Project No. 15 RF-009.

Open Access This article is distributed under the terms of the Creative Commons Attribution 4.0 International License (http://creativecomm ons.org/licenses/by/4.0/), which permits unrestricted use, distribution, and reproduction in any medium, provided you give appropriate credit to the original author(s) and the source, provide a link to the Creative Commons license, and indicate if changes were made. Funded by $\mathrm{SCOAP}^{3}$.

\section{Appendix: Alternative representation of the Hadamard function}

In this section we derive an alternative representation for the Hadamard function which is well suited for the investigation of the near-plate asymptotic of the current density. The starting point is the representation (2.21). We apply to the corresponding series over $n_{l}$ the summation formula $[50,95]$

$$
\begin{aligned}
& \frac{2 \pi}{L_{l}} \sum_{n_{l}=-\infty}^{\infty} g\left(k_{l}\right) f\left(\left|k_{l}\right|\right)=\int_{0}^{\infty} \mathrm{d} u[g(u)+g(-u)] f(u) \\
& +i \int_{0}^{\infty} \mathrm{d} u[f(i u)-f(-i u)] \sum_{\lambda= \pm 1} \frac{g(i \lambda u)}{e^{u L_{l}+i \lambda \tilde{\alpha}_{l}}-1},
\end{aligned}
$$

where $k_{l}$ is given by (2.12). The part in the Hadamard function coming from the first term in the right-hand side of (A.1) coincides with the Hadamard function for 
the geometry of two plates in $D$-dimensional space with topology $R^{p+2} \times T^{q-1}$ and with the lengths of the compact dimensions $\left(L_{p+2}, \ldots, L_{l-1}, L_{l+1}, \ldots, L_{D}\right)$ (the $l$ th dimension is non-compactified). We will denote this function by $G_{R^{p+2} \times T^{q-1}}\left(x, x^{\prime}\right)$. As a result, under the assumption $\beta_{j} \leqslant 0$, the Hadamard function is decomposed as

$$
\begin{aligned}
& G\left(x, x^{\prime}\right)=G_{R^{p+2} \times T^{q-1}}\left(x, x^{\prime}\right)+\frac{L_{l}}{\pi a V_{q}} \int \frac{\mathrm{d} \mathbf{k}_{p}}{(2 \pi)^{p}} \sum_{\mathbf{n}_{q-1}} \\
& \times \sum_{n=1}^{\infty} \frac{\lambda_{n} g\left(z, z^{\prime}, \lambda_{n} / a\right) e^{i \mathbf{k}_{p} \cdot \Delta \mathbf{x}_{p}+i \mathbf{k}_{q-1}^{l} \cdot \Delta \mathbf{x}_{q-1}^{l}}}{\lambda_{n}+\cos \left[\lambda_{n}+2 \tilde{\gamma}_{j}\left(\lambda_{n}\right)\right] \sin \lambda_{n}} \\
& \times \int_{\omega_{\mathbf{k}}^{(l)}}^{\infty} \mathrm{d} u \frac{\cos h\left(\Delta t \sqrt{u^{2}-\omega_{\mathbf{k}}^{(l) 2}}\right)}{\sqrt{u^{2}-\omega_{\mathbf{k}}^{(l) 2}}} \sum_{\lambda= \pm 1} \frac{e^{-\lambda u \Delta x^{l}}}{e^{u L_{l}+i \lambda \tilde{\alpha}_{l}}-1},
\end{aligned}
$$

where $\mathbf{x}_{q-1}^{l}=\left(x^{p+2}, \ldots, x^{l-1}, x^{l+1}, \ldots x^{D}\right), \mathbf{k}_{q-1}=$ $\left(k_{p+2}, \ldots, k_{l-1}, k_{l+1}, \ldots, k_{D}\right)$, and $\omega_{\mathbf{k}}^{(l)}=\sqrt{\omega_{\mathbf{k}}^{2}-k_{l}^{2}}$. Here, the second term in the right-hand side vanishes in the limit $L_{l} \rightarrow \infty$ and is induced by the compactification of the $l$ th dimension from $R^{1}$ to $S^{1}$ with the length $L_{l}$.

By making use of the relation

$$
\sum_{\lambda= \pm 1} \frac{e^{-\lambda u \Delta x^{l}}}{e^{u L_{l}+i \lambda \tilde{\alpha}_{l}}-1}=2 u \sum_{r=1}^{\infty} h_{r}\left(u, \Delta x^{l}\right) \text {, }
$$

with

$$
h_{r}\left(\Delta x^{l}, u\right)=\frac{e^{-r u L_{l}}}{u} \cos h\left(u \Delta x^{l}+i r \tilde{\alpha}_{l}\right) \text {, }
$$

we rewrite the formula (A.2) in the form

$$
\begin{aligned}
& G\left(x, x^{\prime}\right)=G_{R^{p+2} \times T^{q-1}}\left(x, x^{\prime}\right)+\frac{2 L_{l}}{\pi a V_{q}} \sum_{r=1}^{\infty} \int \frac{\mathrm{d} \mathbf{k}_{p}}{(2 \pi)^{p}} \\
& \times \sum_{\mathbf{n}_{q-1}} \int_{0}^{\infty} \mathrm{d} y \cos h(y \Delta t) e^{i \mathbf{k}_{p} \cdot \Delta \mathbf{x}_{p}+i \mathbf{k}_{q-1}^{l} \cdot \Delta \mathbf{x}_{q-1}^{l}} \\
& \times \sum_{n=1}^{\infty} \frac{\lambda_{n} g\left(z, z^{\prime}, \lambda_{n} / a\right) h_{r}\left(\Delta x^{l}, \sqrt{\lambda_{n}^{2} / a^{2}+y^{2}+\omega_{p, \mathbf{n}_{q-1}}^{2}}\right)}{\lambda_{n}+\cos \left[\lambda_{n}+2 \tilde{\gamma}_{j}\left(\lambda_{n}\right)\right] \sin \lambda_{n}},
\end{aligned}
$$

with $\omega_{p, \mathbf{n}_{q-1}}=\sqrt{\mathbf{k}_{p}^{2}+\omega_{\mathbf{n}_{q-1}}^{2}}$. Now, by using the summation formula (2.25) for the series over $n$ we get the final representation

$$
\begin{aligned}
G\left(x, x^{\prime}\right)= & G_{R^{p+2} \times T^{q-1}}\left(x, x^{\prime}\right)+\frac{2 L_{l}}{\pi^{2} V_{q}} \sum_{r=1}^{\infty} \int \frac{\mathrm{d} \mathbf{k}_{p}}{(2 \pi)^{p}} \\
& \times \sum_{\mathbf{n}_{q-1}} e^{i \mathbf{k}_{p} \cdot \Delta \mathbf{x}_{p}+i \mathbf{k}_{q-1}^{l} \cdot \Delta \mathbf{x}_{q-1}^{l}} \\
& \times \int_{0}^{\infty} \mathrm{d} y \cos h(\Delta t y)
\end{aligned}
$$

$$
\begin{aligned}
& \left\{\int_{0}^{\infty} \mathrm{d} u g_{j}\left(z, z^{\prime}, u\right) h_{r}\left(\Delta x^{l}, \sqrt{u^{2}+y^{2}+\omega_{p, \mathbf{n}_{q-1}}^{2}}\right)\right. \\
& +\int_{\sqrt{y^{2}+\omega_{p, \mathbf{n}_{q-1}}^{2}}}^{\infty} \mathrm{d} u \frac{g_{j}\left(z, z^{\prime}, i u\right)}{c_{1}(a u) c_{2}(a u) e^{2 a u}-1} \\
& \left.\times \sum_{s= \pm 1} i h_{s r}\left(\Delta x^{l}, i \sqrt{u^{2}-y^{2}-\omega_{p, \mathbf{n}_{q-1}}^{2}}\right)\right\} .
\end{aligned}
$$

In this expression, the part with the first term in the figure braces is the contribution to the Hadamard function induced by the compactification of the $l$ th dimension for the geometry of a single plate at $x^{p+1}=a_{j}$ and the part with the second term in the figure braces is induced by the second plate. Note that the contribution of the first term in the right-hand side of (A.6) to current density along the $l$ th dimension vanishes.

In deriving the representation (A.6) we have assumed that $\beta_{j} \leqslant 0$. For this case, in the region between the plates, all the eigenvalues for the momentum $k_{p+1}$ are real and in the geometry of a single plate there are no bound states. For $\beta_{j}>0$, in the application of the summation formula (2.25) to the series over $n$ in (A.5) the contribution from the poles $\pm i / b_{j}$ should be added to the right-hand side of (2.25). This contribution comes from the bound state in the geometry of a single plate at $x^{p+1}=a_{j}$. For this bound state the mode function has the form $\varphi_{\mathbf{k}}^{( \pm)}(x) \sim e^{-z_{j} / \beta_{j}} e^{i \mathbf{k}_{\|} \cdot \mathbf{x}_{\|} \mp i \omega_{\mathbf{k}}^{(b)} t}$ with $\omega_{\mathbf{k}}^{(b)}=\sqrt{\mathbf{k}_{p}^{2}+\omega_{\mathbf{n}_{q}}^{2}-1 / \beta_{j}^{2}}$. Assuming that $\omega_{0 l}>1 / \beta_{j}$, the contribution from the bound state to the Hadamard function in the geometry of a single plate is given by the expression

$$
\begin{aligned}
& G_{b j}^{(1)}\left(x, x^{\prime}\right)=\frac{4 \theta\left(\beta_{j}\right) L_{l}}{\pi V_{q} \beta_{j}} e^{-\left|z+z^{\prime}-2 a_{j}\right| / \beta_{j}} \sum_{r=1}^{\infty} \int \frac{\mathrm{d} \mathbf{k}_{p}}{(2 \pi)^{p}} \\
& \times \sum_{\mathbf{n}_{q-1}^{l}} \int_{0}^{\infty} \mathrm{d} x e^{i \mathbf{k}_{p} \cdot \Delta \mathbf{x}_{p}+i \mathbf{k}_{q-1}^{l} \cdot \Delta \mathbf{x}_{q-1}^{l}} \\
& \times \cos h(x \Delta t) h_{r}\left(\Delta x^{l}, \sqrt{x^{2}+\mathbf{k}_{p}^{2}+\omega_{\mathbf{n}_{q-1}}^{2}-1 / \beta_{j}^{2}}\right),
\end{aligned}
$$

where $\theta(x)$ is the Heaviside unit step function. In the case $\omega_{0 l}<1 / \beta_{j}<\omega_{0}$ the corresponding expression is more complicated.

\section{References}

1. V.M. Mostepanenko, N.N. Trunov, The Casimir Effect and its Applications (Clarendon, Oxford, 1997)

2. G. Plunien, B. Müller, W. Greiner, Phys. Rep. 134, 87 (1986)

3. E. Elizalde, S.D. Odintsov, A. Romeo, A.A. Bytsenko, S. Zerbini, Zeta Regularization Techniques with Applications (World Scientific, Singapore, 1994)

4. K.A. Milton, The Casimir Effect: Physical Manifestation of ZeroPoint Energy (World Scientific, Singapore, 2002)

5. M. Bordag, G.L. Klimchitskaya, U. Mohideen, V.M. Mostepanenko, Advances in the Casimir Effect (Oxford University Press, Oxford, 2009) 
6. D. Dalvit, P. Milonni, D. Roberts, F. da Rosa, (eds.), Casimir Physics, Lecture Notes in Physics vol. 834 (Springer, Berlin, 2011)

7. H.B.G. Casimir, Proc. K. Ned. Akad. Wet. 51, 793 (1948)

8. A. Linde, JCAP 0410, 004 (2004)

9. V.P. Gusynin, S.G. Sharapov, J.P. Carbotte, Int. J. Mod. Phys. B 21, 4611 (2007)

10. A.H. Castro Neto, F. Guinea, N.M.R. Peres, K.S. Novoselov, A.K. Geim, Rev. Mod. Phys. 81, 109 (2009)

11. R. Jackiw, S.-Y. Pi, Phys. Rev. Lett. 98, 266402 (2007)

12. O. Oliveira, C.E. Cordeiro, A. Delfino, W. de Paula, T. Frederico, Phys. Rev. B 83, 155419 (2011)

13. M.J. Duff, B.E.W. Nilsson, C.N. Pope, Phys. Rep. 130, 1 (1986)

14. R. Camporesi, Phys. Rep. 196, 1 (1990)

15. A.A. Bytsenko, G. Cognola, L. Vanzo, S. Zerbini, Phys. Rep. 266, 1 (1996)

16. A.A. Bytsenko, G. Cognola, E. Elizalde, V. Moretti, S. Zerbini, Analytic Aspects of Quantum Fields (World Scientific, Singapore, 2003)

17. E. Elizalde, Ten Physical Applications of Spectral Zeta Functions (Springer, Berlin, 2012)

18. F.C. Khanna, A.P.C. Malbouisson, J.M.C. Malbouisson, A.E. Santana, Phys. Rep. 539, 135 (2014)

19. E. Elizalde, Phys. Lett. B 516, 143 (2001)

20. C.L. Gardner, Phys. Lett. B 524, 21 (2002)

21. K.A. Milton, Grav. Cosmol. 9, 66 (2003)

22. A.A. Saharian, Phys. Rev. D 70, 064026 (2004)

23. E. Elizalde, J. Phys. A 39, 6299 (2006)

24. A.A. Saharian, Phys. Rev. D 74, 124009 (2006)

25. B. Green, J. Levin, J. High Energy Phys. 11, 096 (2007)

26. P. Burikham, A. Chatrabhuti, P. Patcharamaneepakorn, K. Pimsamarn, J. High Energy Phys. 07, 013 (2008)

27. P. Chen, Nucl. Phys. B (Proc. Suppl.) 173, s8 (2009)

28. V.M. Mostepanenko, IYu. Sokolov, Phys. Lett. A 125, 405 (1987)

29. J.C. Long, H.W. Chan, J.C. Price, Nucl. Phys. B 539, 23 (1999)

30. R.S. Decca, D. López, E. Fischbach, G.L. Klimchitskaya, D.E. Krause, V.M. Mostepanenko, Ann. Phys. (N.Y.) 318, 37 (2005)

31. R.S. Decca, D. López, E. Fischbach, G.L. Klimchitskaya, D.E. Krause, V.M. Mostepanenko, Phys. Rev. D 75, 077101 (2007)

32. G.L. Klimchitskaya, V.M. Mostepanenko, Eur. Phys. J. C 75, 164 (2015)

33. H.B. Cheng, Phys. Lett. B 643, 311 (2006)

34. H.B. Cheng, Phys. Lett. B 668, 72 (2008)

35. S.A. Fulling, K. Kirsten, Phys. Lett. B 671, 179 (2009)

36. K. Kirsten, S.A. Fulling, Phys. Rev. D 79, 065019 (2009)

37. E. Elizalde, S.D. Odintsov, A.A. Saharian, Phys. Rev. D 79, 065023 (2009)

38. L.P. Teo, Phys. Lett. B 672, 190 (2009)

39. L.P. Teo, Nucl. Phys. B 819, 431 (2009)

40. L.P. Teo, J. High Energy Phys. 11, 095 (2009)

41. K. Poppenhaeger, S. Hossenfelder, S. Hofmann, M. Bleicher, Phys. Lett. B 582, 1 (2004)

42. A. Edery, V.N. Marachevsky, J. High Energy Phys. 12, 035 (2008)

43. F. Pascoal, L.F.A. Oliveira, F.S.S. Rosa, C. Farina, Braz. J. Phys. 38, 581 (2008)

44. L. Perivolaropoulos, Phys. Rev. D 77, 107301 (2008)

45. L.P. Teo, Phys. Rev. D 83, 105020 (2011)

46. S. Bellucci, A.A. Saharian, Phys. Rev. D 80, 105003 (2009)

47. E. Elizalde, S.D. Odintsov, A.A. Saharian, Phys. Rev. D 83, 105023 (2011)

48. F.S. Khoo, L.P. Teo, Phys. Lett. B 703, 199 (2011)

49. M.R. Douglas, S. Kachru, Rev. Mod. Phys. 79, 733 (2007)

50. S. Bellucci, A.A. Saharian, V.M. Bardeghyan, Phys. Rev. D 82, $065011(2010)$

51. S. Bellucci, A.A. Saharian, H.A. Nersisyan, Phys. Rev. D 88, 024028 (2013)
52. E.R. Bezerra de Mello, A.A. Saharian, V. Vardanyan, Phys. Lett. B 741, 155 (2015)

53. E.R. Bezerra de Mello, A.A. Saharian, Phys. Rev. D 87, 045015 (2013)

54. S. Bellucci, E.R. Bezerra de Mello, A.A. Saharian, Phys. Rev. D 89, 085002 (2014)

55. S. Bellucci, A.A. Saharian, Phys. Rev. D 87, 025005 (2013)

56. G. Esposito, A.Y. Kamenshchik, G. Pollifrone, Euclidean Quantum Gravity on Manifolds with Boundary (Springer, Dordrecht, 1997)

57. I.G. Avramidi, G. Esposito, Commun. Math. Phys. 200, 495 (1999)

58. J. Ambjorn, S. Wolfram, Ann. Phys. (N.Y.) 147, 33 (1983)

59. H. Luckock, J. Math. Phys. 32, 1755 (1991)

60. T. Gherghetta, A. Pomarol, Nucl. Phys. B 586, 141 (2000)

61. A. Flachi, D.J. Toms, Nucl. Phys. B 610, 144 (2001)

62. A.A. Saharian, Nucl. Phys. B 712, 196 (2005)

63. S. Bellucci, A.A. Saharian, A.H. Yeranyan, Phys. Rev. D 89, $105006(2014)$

64. S. Bellucci, A.A. Saharian, N.A. Saharyan, Eur. Phys. J. C 74, 3047 (2014)

65. E.R. Bezerra de Mello, A.A. Saharian, Int. J. Theor. Phys. (to appear). arXiv: 1408.6404

66. S.N. Solodukhin, Phys. Rev. D 63, 044002 (2001)

67. C.J. Isham, Proc. R. Soc. A 362, 383 (1978)

68. C.J. Isham, Proc. R. Soc. A 364, 591 (1978)

69. J. Scherk, J.H. Schwartz, Phys. Lett. B 82, 60 (1979)

70. Y. Hosotani, Phys. Lett. B 126, 309 (1983)

71. A. Higuchi, L. Parker, Phys. Rev. D 37, 2853 (1988)

72. Y. Hosotani, Ann. Phys. (N.Y.) 190, 233 (1989)

73. A. Actor, Class. Quantum Grav. 7, 663 (1990)

74. K. Kirsten, J. Phys. A Math. Gen. 26, 2421 (1993)

75. C. Ccapa Ttira, C.D. Fosco, A.P.C. Malbouisson, I. Roditi, Phys. Rev. A 81, 032116 (2010)

76. A. Romeo, A.A. Saharian, J. Phys. A Math. Gen. 35, 1297 (2002)

77. A.A. Saharian, The Generalized Abel-Plana Formula with Applications to Bessel Functions and Casimir Effect (Yerevan State University Publishing House, Yerevan, 2008); Report No. ICTP/2007/082. arXiv:0708.1187

78. D. Deutsch, P. Candelas, Phys. Rev. D 20, 3063 (1979)

79. P. Candelas, Ann. Phys. (N.Y.) 143, 241 (1982)

80. V. Sopova, L.H. Ford, Phys. Rev. D 66, 045026 (2002)

81. N. Graham, R.L. Jaffe, V. Khemani, M. Quandt, M. Scandurra, H. Weigel, Nucl. Phys. B 645, 49 (2002)

82. N. Graham, R.L. Jaffe, H. Weigel, Int. J. Mod. Phys. A 17, 846 (2002)

83. N. Graham, R.L. Jaffe, V. Khemani, M. Quandt, M. Scandurra, H. Weigel, Phys. Lett. B 572, 196 (2003)

84. K.A. Milton, Phys. Rev. D 68, 065020 (2003)

85. K.A. Milton, J. Phys. A Math. Gen. 37, R209 (2004)

86. N. Graham, R.L. Jaffe, V. Khemani, M. Quandt, O. Schröder, H. Weigel, Nucl. Phys. B 677, 379 (2004)

87. K.A. Milton, I. Cavero-Pelaez, J. Wagner, J. Phys. A Math. Gen. 39, 6543 (2006)

88. A.A. Sahariana, Eur. Phys. J. C 52, 721 (2007)

89. K.A. Milton, Phys. Rev. D 84, 065028 (2011)

90. F.D. Mazzitelli, J.P. Nery, A. Satz, Phys. Rev. D 84, 125008 (2011)

91. R. Estrada, S.A. Fulling, L. Kaplan, K. Kirsten, Z. Liu, K.A. Milton, J. Phys. A Math. Theor. 41, 164055 (2008)

92. R. Estrada, S.A. Fulling, F.D. Mera, J. Phys. A Math. Theor. 45, 455402 (2012)

93. N. Bartolo, R. Passante, Phys. Rev. A 86, 012122 (2012)

94. R. Passante, L. Rizzuto, S. Spagnolo, Eur. Phys. J. C 73, 2419 (2013)

95. E.R. Bezerra de Mello, A.A. Saharian, Phys. Rev. D 78, 045021 (2008) 